\title{
Stochastic Analysis of Uplink Interference in Two-Tier Femtocell Networks: Open versus Closed Access
}

\author{
Wei Bao, Student Member, IEEE, and Ben Liang, Senior Member, IEEE
}

\begin{abstract}
We introduce a stochastic analytical framework to compare the performance of open and closed access modes in a two-tier femtocell network, with regard to uplink interference and outage at both the macrocell and femtocell levels. A stochastic geometric approach is employed as the basis for our analysis. We present numerical methods to characterize the distributions of uplink interference and the outage probabilities. We further derive sufficient conditions for open and closed access modes to outperform each other in terms of the outage probability at either the macrocell or femtocell level. This leads to closed-form expressions to upper and lower bound the difference in the targeted received power between the two access modes. Simulations are conducted to validate the accuracy of the analytical model and the correctness of the bounds.
\end{abstract}

Index Terms-Femtocell, uplink interference, stochastic geometry, open access

\section{INTRODUCTION}

In the deployment of wireless cellular networks, some of the most important objectives are to provide higher capacity, better service quality, lower power usage, and ubiquitous coverage. To achieve these goals, one effective approach is to install a second tier of smaller cells, referred to as femtocells, overlapping the original macrocell network [1]. Each femtocell is equipped with a short-range and low-cost base station (BS).

In the presence of femtocells, whenever some User Equipment (UE) is near a femtocell BS, two different access mechanisms may be applied: closed access and open access. Under closed access, a femtocell BS only provides service to its local users, without further admitting nearby macrocell users. In contrast, under open access, all nearby macrocell users are allowed to access the femtocell BS. The open access mode increases the interference level from within a femtocell, but it also allows macrocell UEs that might otherwise transmit at high power toward their faraway macrocell BS to potentially switch to lower-power transmission toward the femtocell BS, therefore reducing the overall interference in the system. However, the relative merits between open access and closed

Manuscript received December 8, 2014; revised April 19, 2015; accepted June 9, 2015. This paper was presented in part at the ACM International Conference on Modeling, Analysis and Simulation of Wireless and Mobile Systems (MSWiM), Barcelona, Spain, November 2013. This work has been supported in part by grants from Bell Canada and the Natural Sciences and Engineering Research Council (NSERC) of Canada. The associate editor coordinating the review of this paper and approving it for publication was Dr. Amine Maaref.

The authors are with the Department of Electrical and Computer Engineering, University of Toronto, Toronto, ON, M5S 3G4, Canada (e-mail: wbao@ ece.utoronto.ca; liang@ece.utoronto.ca). access remain unresolved within the research community, as they may concern diverse factors in communication efficiency, control overhead, system security, and regulatory policies.

In this work, we contribute to the current debate by providing new technical insights on how the two access modes may affect both macrocell users and local femtocell users, in terms of the uplink interference and outage probabilities. We seek to quantify the conditions to guarantee that one access mode improves the performance of macrocell or femtocell users. It is a challenging task, as we need to account for the diverse spatial patterns of different network components. Macrocell BSs are usually deployed regularly by the network operator, while femtocell BSs are spread irregularly, sometimes in an anywhere plug-and-play manner, leading to a high level of spatial randomness. Furthermore, macrocell users are randomly distributed throughout the system, while femtocell users show strong spatial locality and correlation, since they aggregate around femtocell BSs. Whenever open access is applied, we also need to consider the effects of handoffs made by open access users, which brings even more complication to the analytical model.

We present a stochastic geometric analysis framework to derive numerical expressions for the uplink interference and outage probabilities of open access and closed access by modeling macrocell BSs as a regular grid, macrocell UEs as a Poisson point process (PPP), and femtocell UEs as a two-level clustered Poisson point process, which captures the spatial patterns of different network components. Uplink interference analysis is more challenging compared with the downlink case because (1) the spatial patterns of interfering UEs are more complicated, and (2) uplink power control further burdens the analysis by introducing a coupling effect between UEs and BSs. As a result, our analysis yields non-closed forms requiring numerical integrations. This motivates us to further develop closed-form sufficient conditions for open access and closed access to outperform each other, at both the macrocell and femtocell levels.

Based on the above analysis, we are able to extract a key factor that influences the performance difference between open access and closed access: the power enhancement factor $\rho$, which is defined as the ratio, of the uplink targeted received power of an open access user in the femtocell, to its original targeted received power in the macrocell. We investigate the threshold value $\rho^{*}$ (resp. $\rho^{* *}$ ) such that macrocell (resp. femtocell) users may benefit through open access if $\rho<\rho^{*}$ (resp. $\rho<\rho^{* *}$ ) as we apply open access to replace closed 
access. Upper and lower bounds of $\rho^{*}$ are derived in closed forms, and the bounds of $\rho^{* *}$ can be found by numerically searching through closed-form equations, providing system design guidelines with low computational complexity.

The rest of the paper is organized as follows. In Section II, we discuss the relation between our work and prior works. In Section III, we present the system model. In Sections IV and $\mathrm{V}$, we analyze the performance at the macrocell and femtocell levels, respectively. In Section VI, we validate our analysis with simulation results. Finally, concluding remarks are given in Section VII.

\section{RELATED WORKS}

The downlink interference and outage performance in cellular networks have been extensively studied using the stochastic geometric approach. Dhillon et al. [2], [3] analyzed the downlink performance of heterogeneous networks with multiple tiers by assuming the signal-to-interference plus noise ratio (SINR) threshold is greater than 1 . Keeler et al. [4] extended the work by allowing the SINR threshold to be less than 1. Kim et al. [5] studied the maximum tier-1 user and tier-2 cell densities under downlink outage constraints. Dhillon et al. [6] studied the downlink interference considering load balance. Singh et al. [7] studied the downlink user achievable rate in a heterogeneous network considering both SINR and spatial user distributions. Jo et al. [8] studied open access versus closed access in femtocell networks in terms of downlink performance. In the downlink scenario, base station cooperation was studied in [9], [10], where users could access multiple base stations simultaneously.

The analysis of uplink interference in multi-tier networks is more challenging compared with the downlink case. For uplink analysis, the interference generators are the set of UEs, which are clustered and less regularly distributed compared with the interference generators (i.e., BSs) in downlink analysis. Under closed access, without considering random spatial patterns, Kishore et al. [11] studied the uplink performance of a single tier-1 cell and a single femtocell, while the same authors [12] extended it to the case of multiple tier-1 cells and multiple femtocells. An and Pianese [13] studied the cochannel uplink interference in LTE-based multi-tier cellular networks, considering a constant number of femtocells in a macrocell. However, none of [11]-[13] considered the random spatial patterns of users or femtocells.

By considering random spatial patterns, Novlan et al. [14] analyzed the uplink performance of cellular networks, but it was limited to the one-tier case. The work was extended in [15] where frequency reuse among edge users and inner users were accommodated. Chakchouk and Hamdaoui [16] studied the two-tier case with one macrocell and randomly spatially distributed femtocells and femtocell users. Chandrasekhar and Andrews [17] evaluated the uplink performance of two-tier networks considering multiple macrocells, femtocells, macrocell users, and femtocell users. However, several interference components were analyzed based on approximations, such as BSs see a femtocell as a point interference source, and femtocell UEs transmit at the maximum power at the edge of cells. Cheung et al. [18] studied both uplink and downlink interference of femtocell networks based on a Neyman-Scott Process. However, it assumed that each UE transmits at the same power and femtocell UEs are uniformly distributed in an infinitesimally thin ring around the femtocell BS. With a more general system model, Bao and Liang [19] derived the uplink interference in a two-tier network with multiple types of users and small cell BSs, but no closed-form result was obtained. Moreover, the authors of [17]-[19] considered only the closed access case.

The analysis of open access in femtocell networks is even more complicated. This is because the model for open access needs to capture the impact of the users disconnecting from the original macrocell BS and connecting to a femtocell BS. In order to achieve mathematical tractability, the previous analysis of open access used simplified assumptions. Xia et al. [20] compared the performance of open access and closed access based on a model with one macrocell, one femtocell, and a given number of macrocell users, and Tarasak et al. [21] used a model with one macrocell, a constant number of macrocell users, and randomly distributed femtocells. Although [20] and [21] provide useful insights into the performance comparison between open access and closed access, their limited system models cannot account for the challenging issues brought by the diverse spatial patterns of BSs and UEs. Zeinalpour-Yazdi et al. [22] studied a model with one macrocell and randomly spatially distributed macrocell users and femtocells. The model in [22] is still limited to a single-macrocell scenario where the spatial patterns of macrocell BSs and inter-macrocell interference cannot be accommodated. ElSawy and Hossain [23] studied the uplink outage performance of multi-tier cellular networks with truncated channel inversion power control. Open access was considered in [23], but approximations had to be used for mathematical tractability, e.g, the correlation of the locations of interfering UEs and their transmit power levels were ignored. Focusing on a different scope from our work, [23] did not compare the performance of open access and closed access.

Recently, the downlink interference analysis in [7] has been extended to the uplink in [24]. However, there are several differences between our work and [24]. First, in [24], each BS tier (including the macrocell BS tier) is assumed to be Poisson, while in our work, macrocell BSs are assumed to form a deterministic hexagonal point process. Second, in [24], UEs are assumed to be homogeneously distributed, while we generally consider their potential inhomogeneity by assuming that local femtocell UEs aggregate around femtocell BSs. Third, the offloading method considered in [24] is in a different scope compared with our work. In [24], the biased user association scheme is adopted, where each tier of BSs is assigned an association bias value, and UEs are associated with the BS providing the highest biased received power. UEs are offloaded from macrocells to small cells by assigning large association bias values to small cells. In contrast, in our work, we focus on the comparison of the open access and closed access modes. In the open access mode, the macrocell UEs are offloaded to femtocells if they are located inside femtocells. Due to the above differences in system modeling, the resultant 


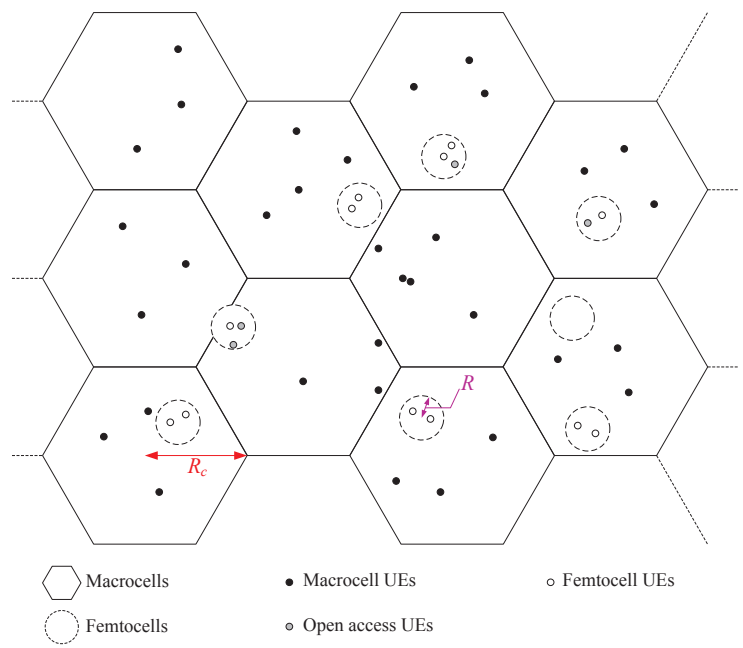

Fig. 1. Two-tier network with macrocells and femtocells.

analysis in our work is substantially different from [24].

Finally, several other works studied the performance of femtocells based on experiments [25], [26], which provide important practical knowledge in designing a real system. Compared with these works, our theoretical approach is an essential alternative that allows more rigorous reasoning to understand the performance benefits of open access compared with closed access, by considering more general system models and behaviors instead of specific experimental scenarios.

A preliminary version of this work was presented in [27]. The current version contains substantial further analytical details, simulation results, and discussion.

\section{System MODEL}

\section{A. Two-Tier Network}

We consider a two-tier network with macrocells and femtocells as shown in Fig. 1. Following a convention in the reserach literature, we assume that the macrocells form an infinite hexagonal grid in the two-dimensional Euclidean space $\mathbb{R}^{2}$. Macrocell BSs are located at the centers of the hexagons $\mathbb{B}=\left\{\left(\frac{3}{2} a R_{c}, \frac{\sqrt{3}}{2} a R_{c}+\sqrt{3} b R_{c}\right) \mid a, b \in \mathbb{Z}\right\}$, where $R_{c}$ is the radius of the hexagon. Macrocell UEs are randomly distributed in the system, which are modeled as a homogeneous Poisson point process (PPP) $\Phi$ with intensity $\lambda$. We focus on interference analysis in a single shared radio frequency sub-band (which is referred to as the reference sub-band) [16], [17], [22], [28], [29]. The UEs (macrocell UEs and femtocell UEs) considered in this work are those using the reference sub-band.

Because femtocell BSs are operated in a plug-and-play fashion, inducing a high level of spatial randomness, we assume femtocell BSs form a homogeneous PPP $\Theta$ with intensity $\mu$. Each femtocell BS is connected to the core network by highcapacity wired links that have no influence on our wireless performance analysis.

Each femtocell BS communicates with local femtocell UEs surrounding it, constituting a femtocell. We assume $R$ as the maximum communication radius of each femtocell BS. Given the location of a femtocell BS at $\mathrm{x}_{0}$, we assume that its femtocell UEs, denoted by $\Psi\left(\mathbf{x}_{0}\right)$, are distributed as a nonhomogenous PPP in the disk centered at $\mathrm{x}_{0}$ with radius $R$. Its intensity at $\mathbf{x}$ is described by $\nu\left(\mathbf{x}-\mathbf{x}_{0}\right)$, a non-negative function of the vector $\mathrm{x}-\mathrm{x}_{0}$. Note that the UE intensity $\nu\left(\mathbf{x}-\mathbf{x}_{0}\right)=0$ if $\left|\mathbf{x}-\mathbf{x}_{0}\right|>R$. The femtocell UEs in one femtocell are independent of femtocell UEs in other femtocells, as well as the macrocell UEs. We assume the scale of femtocells is much smaller than the scale of macrocells [1], $R \ll R_{c}$.

To better understand the spatial distribution of femtocell BSs and femtocell UEs, the femtocell BSs $\Theta$ can be regarded as a parent point process in $\mathbb{R}^{2}$, while femtocell UEs $\Psi$ is a daughter process associated with a point in the parent point process, forming a two-level random pattern (see Section 5.3 of [30]). Note that the aggregating of femtocell UEs around a femtocell BS implicitly defines the location correlation among femtocell UEs.

Let $\mathcal{H}(\mathbf{x})$ denote the hexagon region centered at $\mathbf{x}$ with radius $R_{c}$; let $\mathcal{B}(\mathbf{x}, R)$ denote the disk region centered at $\mathbf{x}$ with radius $R$; let $\mathcal{B S}(\mathbf{x})$ denote the hexagon center nearest to $\mathrm{x}$ (i.e., $\mathcal{B S}(\mathrm{x})=\mathrm{x}_{0} \Leftrightarrow \mathrm{x} \in \mathcal{H}\left(\mathrm{x}_{0}\right)$ ).

\section{B. Open Access versus Closed Access}

If a macrocell UE is covered by a femtocell BS (i.e., within a distance of $R$ from a femtocell $\mathrm{BS}$ ), under closed access, the UE still connects to the macrocell BS. Under open access, the $\mathrm{UE}$ is handed off to the femtocell BS and disconnects from the original macrocell $\mathrm{BS}$; the UE is then referred to as an open access $U E$.

Given a femtocell BS located at $\mathbf{x}_{0}$, let $\Omega\left(\mathbf{x}_{0}\right)$ denote the point process corresponding to the open access UEs connecting to it. We assume that the probability of two femtocells overlapping can be ignored [17]. ${ }^{1}$ Thus, $\Omega\left(\mathbf{x}_{0}\right)$ corresponds to points of $\Phi$ inside the range of the femtocell BS at $\mathbf{x}_{0}$, which is a PPP with intensity $\lambda$ inside $\mathcal{B}\left(\mathrm{x}_{0}, R\right)$.

Let $\bar{\nu}=\int_{\mathcal{B}(\mathbf{0}, R)} \nu(\mathbf{x}) d \mathbf{x}$ be the average number of local femtocell UEs inside a femtocell (using the reference radio frequency sub-band). In the open access case, $\bar{\lambda}=\pi R^{2} \lambda$ is the average number of open access UEs inside a femtocell (using the reference radio frequency sub-band).

\section{Pathloss and Power Control}

Let $P_{t}(\mathbf{x})$ denote the transmission power at $\mathbf{x}$ and $P_{r}(\mathbf{y})$ denote the received power at $\mathbf{y}$. We assume that $P_{r}(\mathbf{y})=$ $\frac{P_{t}(\mathbf{x}) h_{\mathbf{x}, \mathbf{y}}}{|\mathbf{x}-\mathbf{y}|^{\alpha}}$, where $|\mathbf{x}-\mathbf{y}|^{\alpha}$ is the propagation loss function with predetermined constant $\alpha>2$, and $h_{\mathbf{x}, \mathbf{y}}$ is the fast fading term. ${ }^{2}$ Note that we focus on the scenario where different tiers in the system use the same radio spectrum under a similar

\footnotetext{
${ }^{1}$ Given a femtocell, the probability that it does not overlap with another femtocell (i.e., no other femtocell BSs are located $2 R$ from it) is $\mathbb{P}_{\text {nonover }}=$ $\exp \left(-4 \pi R^{2} \mu\right)$. We assume that $R$ and $\mu$ are small enough such that $\mathbb{P}_{\text {nonover }}$ can be approximated by 1 .

${ }^{2}$ In this work, we follow the convention of stochastic geometric analysis (e.g, [3], [7], [14], [22], [23], [31]) where shadowing is ignored. As stated in [31], the main reasons for not considering shadowing in stochastic geometric analysis are (1) shadowing will significantly degrade mathematical tractability in the analysis, and (2) shadowing does not change the main trends of the analytical results.
} 
radio environment, and thus the pathloss exponent values of different tiers are identical [22]-[24]. Also, following a common assumption of stochastic geometric modeling of cellular networks, we assume that $h_{\mathbf{x}, \mathbf{y}}$ is independently exponentially distributed with unit mean (i.e., Rayleigh fading) [3], [7], [14], [22], [23], [31]. Let $H(\cdot)$ be the cumulative distribution function of $h_{\mathbf{x}, \mathbf{y}}$.

We also assume that uplink power control adjusts for propagation losses [17], [28], [32], [33]. The targeted received power level of macrocell UEs, femtocell UEs, and open access UEs are $P, Q$, and $P^{\prime}$, respectively ${ }^{3}$. Given the targeted received power $P_{T}$ (where $P_{T}=P, Q$, or $P^{\prime}$ ), receiver at $\mathbf{y}$, and transmitter at $\mathbf{x}$, the transmission power is $P_{T}|\mathbf{x}-\mathbf{y}|^{\alpha}$. Then, the resultant interference at $\mathbf{y}^{\prime}$ is $\frac{P_{T}|\mathbf{x}-\mathbf{y}|^{\alpha} h_{\mathbf{x}, \mathbf{y}^{\prime}}}{\left|\mathbf{x}-\mathbf{y}^{\prime}\right|^{\alpha}}$.

We define $\rho \triangleq P^{\prime} / P$, which is the targeted received power enhancement if a macrocell UE becomes an open access UE. In this paper, we study the performance variation when open access is applied to replace closed access. Therefore, as a parameter corresponding to open access UEs, $\rho$ is regarded as an important designed parameter. The other parameters, such as $P, Q, T$, and $\alpha$ are considered as predetermined systemlevel constants.

\section{Outage Performance}

In this paper, the performance of macrocell UEs and femtocell UEs (under open access or closed access) is examined through the outage probability, which is defined as the probability that the signal to interference ratio (SIR) is smaller than a given threshold value $T$. Because we focus on interference analysis, the thermal noise is assumed to be negligible.

A partial list of nomenclature is given in Table I.

\section{Open Access vs. Closed Access at the MACROCELL LEVEL}

In this section, we analyze the uplink interference and outage performance of macrocell UEs. Consider a reference macrocell UE, termed the typical UE, communicating with its macrocell BS, termed the typical BS. We aim to investigate the performance of the typical UE.

Due to the stationarity of point processes corresponding to macrocell UEs, femtocell BSs, and femtocell UEs, throughout this section we will re-define the coordinates so that the typical BS is located at $\mathbf{0}$. Correspondingly, the typical UE is located at some $\mathbf{x}_{U}$ that is uniformly distributed in $\mathcal{H}(\mathbf{0})$, since macrocell BSs form a deterministic hexagonal grid (see page 60 of [34]).

Let $\Phi^{\prime}$ be the point process of all other macrocell UEs conditioned on the typical UE, which is a reduced Palm point process [34] with respect to (w.r.t.) $\Phi$. Because the reduced Palm point process of a PPP has the same distribution as its original PPP, $\Phi^{\prime}$ is still a PPP with intensity $\lambda$ [34]. Therefore, for presentation convenience, we still use $\Phi$ to denote this reduced Palm point process.

\footnotetext{
${ }^{3}$ We assume a single fixed level of targeted received power at the macrocell or femtocell level for mathematical tractability. We show that our model is still valid when the targeted received power is randomly distributed through simulations in Section VI.
}

TABLE I

Definition of Selected Variables

\begin{tabular}{|c|c|}
\hline Name & Definition \\
\hline $\mathbb{B}$ & Locations of macrocell BSs \\
\hline$R_{c}$ & Radius of macrocells \\
\hline$\Phi, \lambda$ & Point process and intensity of macrocell UEs \\
\hline$\Theta, \mu$ & Point process and intensity of femtocell BSs \\
\hline$\Psi\left(\mathrm{x}_{0}\right)$ & $\begin{array}{l}\text { Point process of femtocell UEs associating } \\
\text { with a femtocell BS located at } \mathbf{x}_{0}\end{array}$ \\
\hline$\nu(\mathbf{x})$ & $\begin{array}{l}\text { Intensity of femtocell UEs associating } \\
\text { with a femtocell BS. } \mathrm{x} \text { is the relative } \\
\text { coordinate with respect to the femtocell BS }\end{array}$ \\
\hline$\Phi_{0}$ & Points of $\Phi$ not inside any femtocell \\
\hline$\Phi_{1}$ & Points of $\Phi$ inside some femtocell \\
\hline$\Omega\left(\mathbf{x}_{0}\right)$ & $\begin{array}{c}\text { Points of open access UEs } \\
\text { connected to a femtocell BS located at } \mathbf{x}_{0}\end{array}$ \\
\hline$R$ & Radius of femtocells \\
\hline $\begin{array}{c}P, Q \\
P^{\prime}\end{array}$ & $\begin{array}{l}\text { Targeted received powers of macrocell UEs, } \\
\text { femtocell UEs, and open access UEs }\end{array}$ \\
\hline$\rho$ & $P^{\prime} / P$ \\
\hline$\alpha$ & Pathloss exponent \\
\hline$T$ & SIR threshold \\
\hline $\bar{\nu}$ & $\begin{array}{l}\text { Average number of local femtocell UEs } \\
\text { inside a femtocell using the reference } \\
\text { radio frequency sub-band }\end{array}$ \\
\hline $\bar{\lambda}$ & $\begin{array}{l}\text { Average number of open access UEs } \\
\text { inside a femtocell using the reference } \\
\text { radio frequency sub-band }\end{array}$ \\
\hline
\end{tabular}

\section{A. Open Access Case}

1) Interference Components: The overall interference in the uplink has three parts: from macrocell UEs not inside any femtocell (denoted by $I_{1}$ ), from open access UEs (denoted by $I_{2}$ ), and from femtocell UEs (denoted by $I_{3}$ ).

$I_{1}$ can be computed as the sum of interference from each macrocell UE:

$$
I_{1}=\sum_{\mathbf{x} \in \Phi^{0}} \frac{P|\mathbf{x}-\mathcal{B S}(\mathbf{x})|^{\alpha} h_{\mathbf{x}, \mathbf{0}}}{|\mathbf{x}|^{\alpha}}
$$

where $\Phi^{0}$ denotes the points of $\Phi$ not inside any femtocell.

$I_{2}$ can be computed as the sum of interference from all open access UEs of all femtocells:

$$
I_{2}=\sum_{\mathbf{x}_{0} \in \Theta} \sum_{\mathbf{x} \in \Omega\left(\mathbf{x}_{0}\right)} \frac{P^{\prime}\left|\mathbf{x}-\mathbf{x}_{0}\right|^{\alpha} h_{\mathbf{x}, \mathbf{0}}}{|\mathbf{x}|^{\alpha}} .
$$

$I_{3}$ can be computed as the sum of interference from all femtocell UEs of all femtocells:

$$
I_{3}=\sum_{\mathbf{x}_{0} \in \Theta} \sum_{\mathbf{x} \in \Psi\left(\mathbf{x}_{0}\right)} \frac{Q\left|\mathbf{x}-\mathbf{x}_{0}\right|^{\alpha} h_{\mathbf{x}, \mathbf{0}}}{|\mathbf{x}|^{\alpha}} .
$$

The overall interference of open access is $I=I_{1}+I_{2}+I_{3}$. 
2) Laplace Transform of I: In this subsection, we study the Laplace transform of $I$, denoted by $\mathcal{L}_{I}(s)$, which leads to the following theorem:

Theorem 1. Under the open access mode, the Laplace transform of uplink interference at a typical macrocell BS is given by

$$
\begin{gathered}
\mathcal{L}_{I}(s)=\mathbf{E}\left(\prod_{\mathbf{x} \in \Phi} u(\mathbf{x}, s)\right) \cdot \mathbf{E}\left[\prod_{\mathbf{x}_{0} \in \Theta} \frac{\mathbf{E}\left(\prod_{\mathbf{x} \in \Omega\left(\mathbf{x}_{0}\right)} v\left(\mathbf{x}, \mathbf{x}_{0}, s\right)\right)}{\mathbf{E}\left(\prod_{\mathbf{x} \in \Omega\left(\mathbf{x}_{0}\right)} u(\mathbf{x}, s)\right)}\right. \\
\left.\left.\mathbf{E}\left(\prod_{\mathbf{x} \in \Psi\left(\mathbf{x}_{0}\right)} w\left(\mathbf{x}, \mathbf{x}_{0}, s\right)\right)\right)\right]
\end{gathered}
$$

where $u(\mathbf{x}, s) \quad \triangleq \quad \exp \left(-\frac{s P|\mathbf{x}-\mathcal{B S}(\mathbf{x})|^{\alpha} h_{\mathbf{x}, 0}}{|\mathbf{x}|^{\alpha}}\right)$, $v\left(\mathbf{x}, \mathbf{x}_{0}, s\right) \triangleq \exp \left(-\frac{s \rho P\left|\mathbf{x}-\mathbf{x}_{0}\right|^{\alpha} h_{\mathbf{x}, \mathbf{0}}}{|\mathbf{x}|^{\alpha}}\right)$, and $w\left(\mathbf{x}, \mathbf{x}_{0}, s\right) \triangleq$ $\exp \left(-\frac{s Q\left|\mathbf{x}-\mathbf{x}_{0}\right|^{\alpha} h_{\mathbf{x}, \mathbf{0}}}{|\mathbf{x}|^{\alpha}}\right)$.

Proof: See Appendix-A for the proof.

3) Numeric Computation of $\mathcal{L}_{I}(s)$ : In this subsection, we present a numeric approach to compute $\mathcal{L}_{I}(s)$ derived in (4), which will facilitate later comparison between open access and closed access. Let $\mathcal{L}_{0}(s)=\mathbf{E}\left(\prod_{x \in \Phi} u(\mathbf{x}, s)\right)$, which is a generating functional corresponding to $\Phi$ [30], [34]. It can be re-written in a standard integral form as follows:

$$
\begin{aligned}
\mathcal{L}_{0}(s) & =\exp \left(-\lambda \int_{\mathbb{R}^{2}}\left(1-\int_{\mathbb{R}+} e^{-\frac{s P|\mathbf{x}-\mathcal{B S}(\mathbf{x})|^{\alpha} h}{|\mathbf{x}|^{\alpha}}} H(d h)\right) d \mathbf{x}\right) \\
& =\exp \left(-\lambda \int_{\mathbb{R}^{2}} \frac{\frac{s P|\mathbf{x}-\mathcal{B S}(\mathbf{x})|^{\alpha}}{|\mathbf{x}|^{\alpha}}}{\frac{s P|\mathbf{x}-\mathcal{B S}(\mathbf{x})|^{\alpha}}{|\mathbf{x}|^{\alpha}}+1} d \mathbf{x}\right) .
\end{aligned}
$$

Given the location of a femtocell BS at $\mathbf{x}_{0}$, let $\mathcal{W}\left(\mathbf{x}_{0}, s\right)=$ $\mathbf{E}\left(\prod_{\mathbf{x} \in \Psi\left(\mathbf{x}_{0}\right)} w\left(\mathbf{x}, \mathbf{x}_{0}, s\right)\right)$, which is a generating functional corresponding to $\Psi\left(\mathbf{x}_{0}\right)$. It can be expressed in a standard form through the Laplace functional of PPP $\Psi\left(\mathrm{x}_{0}\right)$ :

$$
\mathcal{W}\left(\mathbf{x}_{0}, s\right)=\exp \left(-\int_{\mathcal{B}(\mathbf{0}, R)} \frac{\frac{s Q|\mathbf{x}|^{\alpha}}{\left|\mathbf{x}+\mathbf{x}_{0}\right|^{\alpha}}}{\frac{s Q|\mathbf{x}|^{\alpha}}{\left|\mathbf{x}+\mathbf{x}_{0}\right|^{\alpha}}+1} \nu(\mathbf{x}) d \mathbf{x}\right) \text {. }
$$

Similarly, let $\mathcal{V}\left(\mathbf{x}_{0}, s\right)=\mathbf{E}\left(\prod_{\mathbf{x} \in \Omega\left(\mathbf{x}_{0}\right)} v\left(\mathbf{x}, \mathbf{x}_{0}, s\right)\right)$, and $\mathcal{U}\left(\mathbf{x}_{0}, s\right)=\mathbf{E}\left(\prod_{\mathbf{x} \in \Omega\left(\mathbf{x}_{0}\right)} u(\mathbf{x}, s)\right)$, we have

$$
\begin{aligned}
& \mathcal{V}\left(\mathbf{x}_{0}, s\right)=\exp \left(-\lambda \int_{\mathcal{B}(\mathbf{0}, R)} \frac{\frac{s \rho P|\mathbf{x}|^{\alpha}}{\left|\mathbf{x}+\mathbf{x}_{0}\right|^{\alpha}}}{\frac{s \rho P|\mathbf{x}|^{\alpha}}{\left|\mathbf{x}+\mathbf{x}_{0}\right|^{\alpha}}+1} d \mathbf{x}\right) \\
& \mathcal{U}\left(\mathbf{x}_{0}, s\right)=\exp \left(-\lambda \int_{\mathcal{B}\left(\mathbf{x}_{0}, R\right)} \frac{\frac{s P|\mathbf{x}-\mathcal{B S}(\mathbf{x})|^{\alpha}}{|\mathbf{x}|^{\alpha}}}{\frac{s P|\mathbf{x}-\mathcal{B S}(\mathbf{x})|^{\alpha}}{|\mathbf{x}|^{\alpha}}+1} d \mathbf{x}\right)
\end{aligned}
$$

Let $\mathcal{J}\left(\mathbf{x}_{0}, s\right)=\frac{\mathcal{V}\left(\mathbf{x}_{0}, s\right)}{\mathcal{U}\left(\mathbf{x}_{0}, s\right)} \mathcal{W}\left(\mathbf{x}_{0}, s\right)$, which is numerically computable through (6)-(8). Finally, we note that

$$
\left.\mathbf{E}\left[\prod_{\mathbf{x}_{0} \in \Theta} \frac{\mathbf{E}\left(\prod_{\mathbf{x} \in \Omega\left(\mathbf{x}_{0}\right)} v\left(\mathbf{x}, \mathbf{x}_{0}, s\right)\right)}{\mathbf{E}\left(\prod_{\mathbf{x} \in \Omega\left(\mathbf{x}_{0}\right)} u(\mathbf{x}, s)\right)} \mathbf{E}\left(\prod_{\mathbf{x} \in \Psi\left(\mathbf{x}_{0}\right)} w\left(\mathbf{x}, \mathbf{x}_{0}, s\right)\right)\right)\right]
$$

$$
\begin{aligned}
& =\mathbf{E}\left[\prod_{\mathbf{x}_{0} \in \Theta}\left(\frac{\mathcal{V}\left(\mathbf{x}_{0}, s\right)}{\mathcal{U}\left(\mathbf{x}_{0}, s\right)} \mathcal{W}\left(\mathbf{x}_{0}, s\right)\right)\right]=\mathbf{E}\left(\prod_{\mathbf{x}_{0} \in \Theta} \mathcal{J}\left(\mathbf{x}_{0}, s\right)\right) \\
& =\exp \left(-\mu \int_{\mathbb{R}^{2}}\left(1-\mathcal{J}\left(\mathbf{x}_{0}, s\right)\right) d \mathbf{x}_{0}\right)
\end{aligned}
$$

where (9) is derived from the generating functional with respect to PPP $\Theta$. Substituting (5) and (9) into (4), we can numerically compute $\mathcal{L}_{I}(s)$ :

$$
\mathcal{L}_{I}(s)=\mathcal{L}_{0}(s) \cdot \exp \left(-\mu \int_{\mathbb{R}^{2}}\left(1-\mathcal{J}\left(\mathbf{x}_{0}, s\right)\right) d \mathbf{x}_{0}\right) .
$$

An intuitive explanation to the above is as follows. First, in terms of the Laplace transform, additive interference is in the product form, and interference reduction is in the division form. Suppose that there are no femtocells at the beginning, and $\mathcal{L}_{0}(s)$ corresponds to the interference from macrocell UEs. Then, we add femtocells to the system. Given a femtocell BS at $\mathbf{x}_{0}, \mathcal{W}\left(\mathbf{x}_{0}, s\right)$ corresponds to the interference from local femtocell UEs inside the femtocell, $\mathcal{V}\left(\mathbf{x}_{0}, s\right)$ corresponds to interference from open access UEs inside the femtocell, and $\mathcal{U}\left(\mathrm{x}_{0}, s\right)$ corresponds to interference reduction due to open access UEs as they disconnect from their original macrocell BS. Thus, $\mathcal{J}\left(\mathbf{x}_{0}, s\right)=\frac{\mathcal{V}\left(\mathbf{x}_{0}, s\right)}{\mathcal{U}\left(\mathbf{x}_{0}, s\right)} \mathcal{W}\left(\mathbf{x}_{0}, s\right)$ represents the overall interference variation when a femtocell centered at $\mathbf{x}_{0}$ is added. Finally, $\exp \left(-\mu \int_{\mathbb{R}^{2}}\left(1-\mathcal{J}\left(\mathbf{x}_{0}, s\right)\right) d \mathbf{x}_{0}\right)$ is the overall interference variation after adding all femtocells. As a consequence, the overall interference can be computed in formula (10).

4) Outage Probability: Given the SIR threshold $T$, the outage probability of the typical UE can be computed as the probability that the signal strength $P h_{\mathbf{x}_{U}, 0}$ over the interference $I$ is less than $T$ :

$$
P_{\text {out }}^{o}=\mathbf{P}\left(P h_{\mathbf{x}_{U}, \mathbf{0}}<T I\right)=1-\left.\mathcal{L}_{I}(s)\right|_{s=\frac{T}{P}} .
$$

The last equality above is due to $h_{\mathbf{x}_{U}, \mathbf{0}}$ being exponentially distributed with unit mean. As a result, $P_{\text {out }}^{o}$ can be derived directly from $\mathcal{L}_{I}(s)$ (see Section 16.2.2.1 of [34]).

\section{B. Closed Access Case}

Different from the open access case, the overall interference has only two parts: from macrocell UEs (denoted by $\widehat{I}_{1}$ ) and from femtocell UEs (denoted by $\widehat{I}_{3}$ ).

$\widehat{I_{1}}$ can be computed as the sum of interference from each macrocell UE:

$$
\widehat{I}_{1}=\sum_{\mathbf{x} \in \Phi} \frac{P|\mathbf{x}-\mathcal{B S}(\mathbf{x})|^{\alpha} h_{\mathbf{x}, \mathbf{0}}}{|\mathbf{x}|^{\alpha}} .
$$

$\widehat{I}_{3}$ is exactly the same as $I_{3}$ in (3).

Then, the total interference can be computed as $\widehat{I}=\widehat{I}_{1}+\widehat{I}_{3}$. Similar to Section IV-A3, the Laplace transform of $\widehat{I}$ is

$$
\begin{aligned}
\mathcal{L}_{\widehat{I}}(s) & =\mathbf{E}\left[\prod_{\mathbf{x} \in \Phi} u(\mathbf{x}, s) \prod_{\mathbf{x}_{0} \in \Theta} \prod_{\mathbf{x} \in \Psi\left(\mathbf{x}_{0}\right)} w\left(\mathbf{x}, \mathbf{x}_{0}, s\right)\right] \\
& =\mathcal{L}_{0}(s) \cdot \mathbf{E}\left[\prod_{\mathbf{x}_{0} \in \Theta}\left(\mathcal{W}\left(\mathbf{x}_{0}, s\right)\right)\right]
\end{aligned}
$$




$$
=\mathcal{L}_{0}(s) \cdot \exp \left(-\mu \int_{\mathbb{R}^{2}}\left(1-\mathcal{W}\left(\mathbf{x}_{0}, s\right)\right) d \mathbf{x}_{0}\right),
$$

where $\mathcal{L}_{0}(s)$ is the same as (5), and $\mathcal{W}\left(\mathbf{x}_{0}, s\right)$ is the same as (6).

An intuitive explanation to the above is as follows. First, $\mathcal{L}_{0}(s)$ corresponds to the interference of all macrocell UEs. Given a femtocell BS at $\mathbf{x}_{0}, \mathcal{W}\left(\mathbf{x}_{0}, s\right)$ corresponds to interference from local femtocell UEs inside the femtocell. Then, $\exp \left(-\mu \int_{\mathbb{R}^{2}}\left(1-\mathcal{W}\left(\mathbf{x}_{0}, s\right)\right) d \mathbf{x}_{0}\right)$ is the overall interference from all femtocells. As a consequence, the overall interference can be computed as formula (13).

Finally, the outage probability of the typical UE can be computed as

$$
P_{\text {out }}^{c}=\mathbf{P}\left(P h_{\mathbf{x}_{U}, \mathbf{0}}<T \widehat{I}\right)=1-\left.\mathcal{L}_{\widehat{I}}(s)\right|_{s=\frac{T}{P}} .
$$

\section{Parameter Normalization}

From the above performance analysis of both open access and closed access, we see that one can normalize the radius of macrocells $R_{c}$ to 1 , so that $R$ is equivalent to the ratio of the radius of femtocells to that of macrocells $(R \ll 1)$. Also, we can normalize the target received power of macrocell UEs $P$ to 1 , so that $Q$ is equivalent to the ratio of the target received power of femtocell UEs to that of macrocell UEs, and $P^{\prime}=\rho$. Therefore, in the rest of this section, without loss of generality, we set $R_{c}=1$ and $P=1$.

\section{Open Access vs. Closed Access}

We compare the outage performance of open access and closed access at the macrocell level. Due to the integral form of the Laplace transform, the expressions of outage probabilities for both the open and closed access cases are in non-closed forms, requiring multiple levels of integration. As a consequence, we are motivated to derive closed-form bounds to compare open access and closed access.

Let $\mathbf{V}_{\max } \triangleq 4 \pi^{2} R^{4}(T \rho)^{\frac{2}{\alpha}}\left(\frac{1}{8}+\frac{1}{4(\alpha+2)}+\frac{1}{(\alpha+2)(\alpha-2)}\right)$, $\mathbf{V}_{\text {min }} \triangleq 2 \pi^{2} R^{4}(T \rho)^{\frac{2}{\alpha}}\left(\frac{1}{8}+\frac{1}{4(\alpha+2)}+\frac{1}{(\alpha+2)(\alpha-2)}\right)$, and $C_{u} \triangleq \int_{\mathbb{R}^{2}}\left(\frac{\frac{T|\mathbf{x}-\mathcal{B S}(\mathbf{x})|^{\alpha}}{\mid \mathbf{x}^{\alpha}}}{\frac{T|\mathbf{x} \mathcal{B} \mathcal{S}(\mathbf{x})|^{\alpha}}{|\mathbf{x}|^{\alpha}}+1}\right) d \mathbf{x}$ be a system-level constant determined by $T$ and $\alpha$. We have the following theorem:

Theorem 2. A sufficient condition for $P_{\text {out }}^{o}<P_{\text {out }}^{c}$ is

$$
-\mathbf{V}_{\max }+\pi R^{2} C_{u} e^{-\bar{\nu}}>0,
$$

and a sufficient condition for $P_{\text {out }}^{o}>P_{\text {out }}^{c}$ is

$$
-\pi R^{2} C_{u} e^{\bar{\lambda}}+\mathbf{V}_{\min } e^{-\bar{\lambda}-\bar{\nu}}>0 .
$$

Proof: See Appendix-B for the proof.

Through Theorem 2, closed-form expressions can be used to compare the outage probabilities between open access and closed access without the computational complexity introduced by numeric integrations in (10) and (13).

In the following, we focus on the performance variation if open access is applied to replace closed access. The parameter corresponding to open access UEs, $\rho$, is regarded as a designed parameter. If we fix all the other network parameters, increasing $\rho$ implies better performance for open access UEs, but it will also increase the interference from open access UEs to macrocell BSs. As a consequence, we aim to derive the threshold $\rho^{*}$ such that $P_{\text {out }}^{o}=P_{\text {out }}^{c}$. At the macrocell level, macrocell UEs experience less outage iff $\rho<\rho^{*}$. Thus, $\rho^{*}$ is referred to as the maximum power enhancement tolerated at the macrocell level. Thus, in the deployment of open access femtocells, the network operator is motivated to limit $\rho$ below $\rho^{*}$ to guarantee that the performance of macrocell UEs under open access is no worse than that under closed access. One way to derive $\rho^{*}$ is through numerical computation of (10) and (13) and numerical search, which introduces high computational complexity due to the multiple levels of integration. A more efficient alternative is to find the bounds of $\rho^{*}$ through Theorem 2. Simple algebra manipulation leads to

$$
\begin{aligned}
& \rho_{\text {min }}^{*}=\frac{1}{T}\left(\frac{C_{u} e^{-\bar{\nu}}}{4 \pi R^{2}\left(\frac{1}{8}+\frac{1}{4(\alpha+2)}+\frac{1}{(\alpha+2)(\alpha-2)}\right)}\right)^{\frac{\alpha}{2}}, \\
& \rho_{\max }^{*}=\frac{1}{T}\left(\frac{C_{u} e^{\bar{\nu}+2 \bar{\lambda}}}{2 \pi R^{2}\left(\frac{1}{8}+\frac{1}{4(\alpha+2)}+\frac{1}{(\alpha+2)(\alpha-2)}\right)}\right)^{\frac{\alpha}{2}},
\end{aligned}
$$

where $\rho_{\min }^{*}$ and $\rho_{\max }^{*}$ are the lower bound and upper bound of $\rho^{*}$, respectively. If the network operator limits $\rho<\rho_{\min }^{*}$, the performance of macrocell UEs under open access can be guaranteed no worse than their performance under closed access.

Furthermore, through (17) and (18), we observe that $\rho_{\min }^{*}=$ $\Theta\left(\frac{1}{R^{\alpha}}\right)$ and $\rho_{\max }^{*}=\Theta\left(\frac{1}{R^{\alpha}}\right)$, leading to the following corollary:

\section{Corollary 1.}

$$
\rho^{*}=\Theta\left(\frac{1}{R^{\alpha}}\right) .
$$

Note that, in (19), $R_{c}$ is normalized to 1 and $R$ represents the ratio of the radius of femtocells to that of macrocells. If $R_{c}$ is not normalized, (19) should be re-written as $\rho^{*}=$ $\Theta\left(\left(\frac{R_{c}}{R}\right)^{\alpha}\right)$.

Intuitively, as a rough estimation, open access UEs have their distance to the BS reduced approximately by a factor of $R$, leading to the capability to increase their received power by the corresponding gain in the propagation loss function, as their average interference level is maintained. However, Corollary 1 cannot be trivially obtained from the above intuition. This is because the outage probability does not only depend on the average interference, but also depends on the distribution of the interference (i.e., the Laplace transform of the interference). Comparing (10) with (13), we note that if we switch from closed access to open access, the distribution of the interference will change drastically. Corollary 1 can be derived only after rigorously comparing and bounding the Laplace transforms of interference under open access and closed access.

From (17) and (18), the gap between the upper and lower bounds can be expressed as $\frac{\rho_{\max }^{*}}{\rho_{\min }^{*}}=2^{\frac{\alpha}{2}} e^{\alpha(\bar{\nu}+\bar{\lambda})}$. We emphasize that in this work, interference analysis is conducted for a single shared radio frequency sub-band (i.e., the reference sub-band). 
The average number of UEs in a macrocell or femtocell using the reference sub-band is usually no greater than one. Note that $\bar{\nu}+\bar{\lambda}$ is the average number of UEs in a femtocell using the reference sub-band and is expected to typically be a small value. For example, as indicated in the 3GPP guidelines in Annex A of [35], the number of femtocell UEs per femtocell is much smaller than the number of macrocell UEs per macrocell. Therefore, $\frac{\rho_{\max }^{*}}{\rho_{\min }^{*}}$ is expected to be small in practical systems.

\section{Open Access vs. Closed Access at the FEMTOCELL LEVEL}

In this section, we analyze the uplink interference and outage performance of femtocell UEs. Given a reference femtocell UE, termed the typical femtocell UE, communicating with its femtocell BS, termed the typical femtocell BS, we aim to study the interference at the typical femtocell BS. We also define the femtocell corresponding to the typical femtocell BS as the typical femtocell, and the macrocell BS nearest to the typical femtocell BS as the typical macrocell BS.

Similarly to Section IV, we re-define the coordinate of the typical macrocell BS as 0. Correspondingly, the typical femtocell BS is located at some $\mathbf{x}_{B}$ that is uniformly distributed in $\mathcal{H}(\mathbf{0})$ [34]. Given the typical femtocell centered at $\mathbf{x}_{B}$, let $\Theta^{\prime}$ denote the point process of other femtocell BSs conditioned on the typical femtocell BS, i.e., the reduced Palm point process w.r.t. $\Theta$. Then, $\Theta^{\prime}$ is still a PPP with intensity $\mu$ [34]. For presentation convenience, we still use $\Theta$ to denote this reduced Palm point process. Let $\widetilde{\Psi}\left(\mathbf{x}_{B}\right)$ denote the other femtocell UEs inside the typical femtocell conditioned on the typical femtocell UE. Similarly, $\widetilde{\Psi}\left(\mathbf{x}_{B}\right)$ has the same distribution as $\Psi\left(\mathbf{x}_{B}\right)$. Let $\widetilde{\Omega}\left(\mathbf{x}_{B}\right)$ denote open access UEs connecting to the typical femtocell BS.

\section{A. Open Access Case}

1) Interference Components: The overall interference in the uplink of the typical femtocell UE has five parts: from macrocell UEs not inside any femtocell $\left(I_{1}^{\prime}\left(\mathbf{x}_{B}\right)\right)$, from open access UEs outside the typical femtocell $\left(I_{2}^{\prime}\left(\mathbf{x}_{B}\right)\right)$, from femtocell UEs outside the typical femtocell $\left(I_{3}^{\prime}\left(\mathbf{x}_{B}\right)\right)$, from local femtocell UEs inside the typical femtocell $\left(I_{4}^{\prime}\left(\mathbf{x}_{B}\right)\right)$, and from open access UEs inside the typical femtocell $\left(I_{5}^{\prime}\left(\mathbf{x}_{B}\right)\right)$. We have

$$
\begin{aligned}
& I_{1}^{\prime}\left(\mathbf{x}_{B}\right)=\sum_{\mathbf{x} \in \Phi^{0}} \frac{P|\mathbf{x}-\mathcal{B S}(\mathbf{x})|^{\alpha} h_{\mathbf{x}, \mathbf{x}_{B}}}{\left|\mathbf{x}-\mathbf{x}_{B}\right|^{\alpha}} \\
& I_{2}^{\prime}\left(\mathbf{x}_{B}\right)=\sum_{\mathbf{x}_{0} \in \Theta} \sum_{\mathbf{x} \in \Omega\left(\mathbf{x}_{0}\right)} \frac{\rho P\left|\mathbf{x}-\mathbf{x}_{0}\right|^{\alpha} h_{\mathbf{x}, \mathbf{x}_{B}}}{\left|\mathbf{x}-\mathbf{x}_{B}\right|^{\alpha}}, \\
& I_{3}^{\prime}\left(\mathbf{x}_{B}\right)=\sum_{\mathbf{x}_{0} \in \Theta} \sum_{\mathbf{x} \in \Psi\left(\mathbf{x}_{0}\right)} \frac{Q\left|\mathbf{x}-\mathbf{x}_{0}\right|^{\alpha} h_{\mathbf{x}, \mathbf{x}_{B}}}{\left|\mathbf{x}-\mathbf{x}_{B}\right|^{\alpha}}, \\
& I_{4}^{\prime}\left(\mathbf{x}_{B}\right)=\sum_{\mathbf{x} \in \widetilde{\Psi}\left(\mathbf{x}_{B}\right)} Q h_{\mathbf{x}, \mathbf{x}_{B}}, \\
& I_{5}^{\prime}\left(\mathbf{x}_{B}\right)=\sum_{\mathbf{x} \in \widetilde{\Omega}\left(\mathbf{x}_{B}\right)} \rho P h_{\mathbf{x}, \mathbf{x}_{B}} .
\end{aligned}
$$

The overall interference is $I^{\prime}\left(\mathbf{x}_{B}\right)=\sum_{i=1}^{5} I_{i}^{\prime}\left(\mathbf{x}_{B}\right)$.
2) Laplace Transform of $I^{\prime}\left(\mathbf{x}_{B}\right)$ : In this subsection, we study the Laplace transform of $I^{\prime}\left(\mathbf{x}_{B}\right)$, denoted by $\mathcal{L}_{I^{\prime}}\left(\mathbf{x}_{B}, s\right)$. We have the following theorem:

Theorem 3. Under the open access mode, the Laplace transform of uplink interference at a typical femtocell BS located at $\mathbf{x}_{B}$ is given by

$$
\begin{aligned}
& \mathcal{L}_{I^{\prime}}\left(\mathbf{x}_{B}, s\right)=\mathbf{E}\left(\prod_{\mathbf{x} \in \Phi} u^{\prime}\left(\mathbf{x}, \mathbf{x}_{B}, s\right)\right) \\
& \mathbf{E}\left[\prod_{\mathbf{x}_{0} \in \Theta}\left(\frac{\mathbf{E}\left(\prod_{\mathbf{x} \in \Omega\left(\mathbf{x}_{0}\right)} v^{\prime}\left(\mathbf{x}, \mathbf{x}_{0}, \mathbf{x}_{B}, s\right)\right)}{\mathbf{E}\left(\prod_{\mathbf{x} \in \Omega\left(\mathbf{x}_{0}\right)} u^{\prime}\left(\mathbf{x}, \mathbf{x}_{B}, s\right)\right)} \mathbf{E}\left(\prod_{\mathbf{x} \in \Psi\left(\mathbf{x}_{0}\right)} w^{\prime}\left(\mathbf{x}, \mathbf{x}_{0}, \mathbf{x}_{B}, s\right)\right)\right)\right] \\
& \mathbf{E}\left(\prod_{\mathbf{x} \in \widetilde{\Psi}\left(\mathbf{x}_{B}\right)} w^{\prime}\left(\mathbf{x}, \mathbf{x}_{B}, \mathbf{x}_{B}, s\right)\right) \frac{\mathbf{E}\left(\prod_{\mathbf{x} \in \widetilde{\Omega}\left(\mathbf{x}_{B}\right)} v^{\prime}\left(\mathbf{x}, \mathbf{x}_{B}, \mathbf{x}_{B}, s\right)\right)}{\mathbf{E}\left(\prod_{\mathbf{x} \in \widetilde{\Omega}\left(\mathbf{x}_{B}\right)} u^{\prime}\left(\mathbf{x}, \mathbf{x}_{B}, s\right)\right)}
\end{aligned}
$$

where $u^{\prime}\left(\mathbf{x}, \mathbf{x}_{B}, s\right) \triangleq \quad \exp \left(-\frac{s P|\mathbf{x}-\mathcal{B S}(\mathbf{x})|^{\alpha} h_{\mathbf{x}, \mathbf{x}_{B}}}{\left|\mathbf{x}-\mathbf{x}_{B}\right|^{\alpha}}\right)$, $v^{\prime}\left(\mathbf{x}, \mathbf{x}_{0}, \mathbf{x}_{B}, s\right) \triangleq \exp \left(-\frac{s \rho P\left|\mathbf{x}-\mathbf{x}_{0}\right|^{\alpha} h_{\mathbf{x}, \mathbf{x}_{B}}}{\left|\mathbf{x}-\mathbf{x}_{B}\right|^{\alpha}}\right)$, and $w^{\prime}\left(\mathbf{x}, \mathbf{x}_{0}, \mathbf{x}_{B}, s\right) \triangleq \exp \left(-\frac{s Q\left|\mathbf{x}-\mathbf{x}_{0}\right|^{\alpha} h_{\mathbf{x}, \mathbf{x}_{B}}}{\left|\mathbf{x}-\mathbf{x}_{B}\right|^{\alpha}}\right)$.

Proof: See Appendix-C for the proof.

3) Numeric Computation of $\mathcal{L}_{I^{\prime}}\left(\mathbf{x}_{B}, s\right)$ : First, similar to the derivations of (5)-(8) in Section IV-A3, we have

$$
\begin{aligned}
& \mathcal{L}_{0}^{\prime}\left(\mathbf{x}_{B}, s\right)=\mathbf{E}\left(\prod_{\mathbf{x} \in \Phi} u^{\prime}\left(\mathbf{x}, \mathbf{x}_{B}, s\right)\right) \\
& =\exp \left(-\lambda \int_{\mathbb{R}^{2}} \frac{\frac{s P|\mathbf{x}-\mathcal{B S}(\mathbf{x})|^{\alpha}}{\left|\mathbf{x}-\mathbf{x}_{B}\right|^{\alpha}}}{\frac{s P|\mathbf{x}-\mathcal{B S}(\mathbf{x})|^{\alpha}}{\left|\mathbf{x}-\mathbf{x}_{B}\right|^{\alpha}}+1} d \mathbf{x}\right), \\
& \mathcal{W}^{\prime}\left(\mathbf{x}_{0}, \mathbf{x}_{B}, s\right)=\mathbf{E}\left(\prod_{\mathbf{x} \in \Psi\left(\mathbf{x}_{0}\right)} w^{\prime}\left(\mathbf{x}, \mathbf{x}_{0}, \mathbf{x}_{B}, s\right)\right) \\
& =\exp \left(-\int_{\mathcal{B}\left(\mathbf{x}_{0}, R\right)} \frac{\frac{s Q\left|\mathbf{x}-\mathbf{x}_{0}\right|^{\alpha}}{\left|\mathbf{x}-\mathbf{x}_{B}\right|^{\alpha}}}{\frac{s Q\left|\mathbf{x}-\mathbf{x}_{0}\right|^{\alpha}}{\left|\mathbf{x}-\mathbf{x}_{B}\right|^{\alpha}}+1} \nu\left(\mathbf{x}-\mathbf{x}_{0}\right) d \mathbf{x}\right), \\
& \mathcal{V}^{\prime}\left(\mathbf{x}_{0}, \mathbf{x}_{B}, s\right)=\mathbf{E}\left(\prod_{\mathbf{x} \in \Omega\left(\mathbf{x}_{0}\right)} v^{\prime}\left(\mathbf{x}, \mathbf{x}_{0}, \mathbf{x}_{B}, s\right)\right) \\
& =\exp \left(-\lambda \int_{\mathcal{B}\left(\mathbf{x}_{0}, R\right)} \frac{\frac{s \rho P\left|\mathbf{x}-\mathbf{x}_{0}\right|^{\alpha}}{\left|\mathbf{x}-\mathbf{x}_{B}\right|^{\alpha}}}{\frac{s \rho P\left|\mathbf{x}-\mathbf{x}_{0}\right|^{\alpha}}{\left|\mathbf{x}-\mathbf{x}_{B}\right|^{\alpha}}+1} d \mathbf{x}\right), \\
& \mathcal{U}^{\prime}\left(\mathbf{x}_{0}, \mathbf{x}_{B}, s\right)=\mathbf{E}\left(\prod_{\mathbf{x} \in \Omega\left(\mathbf{x}_{0}\right)} u^{\prime}\left(\mathbf{x}, \mathbf{x}_{B}, s\right)\right) \\
& =\exp \left(-\lambda \int_{\mathcal{B}\left(\mathbf{x}_{0}, R\right)} \frac{\frac{s P|\mathbf{x}-\mathcal{B S}(\mathbf{x})|^{\alpha}}{\left|\mathbf{x}-\mathbf{x}_{B}\right|^{\alpha}}}{\frac{s P|\mathbf{x}-\mathcal{B S}(\mathbf{x})|^{\alpha}}{\left|\mathbf{x}-\mathbf{x}_{B}\right|^{\alpha}}+1} d \mathbf{x}\right),
\end{aligned}
$$

In addition, we can derive

$$
\begin{aligned}
& \mathcal{W}^{\prime \prime}\left(\mathbf{x}_{B}, s\right)=\mathbf{E}\left(\prod_{\mathbf{x} \in \widetilde{\Psi}\left(\mathbf{x}_{B}\right)} w^{\prime}\left(\mathbf{x}, \mathbf{x}_{B}, \mathbf{x}_{B}, s\right)\right) \\
= & \exp \left(-\frac{s Q \bar{\nu}}{s Q+1}\right), \\
& \mathcal{V}^{\prime \prime}\left(\mathbf{x}_{B}, s\right)=\mathbf{E}\left(\prod_{\mathbf{x} \in \widetilde{\Omega}\left(\mathbf{x}_{B}\right)} v^{\prime}\left(\mathbf{x}, \mathbf{x}_{B}, \mathbf{x}_{B}, s\right)\right)
\end{aligned}
$$




$$
\begin{aligned}
= & \exp \left(-\frac{s \rho P \bar{\lambda}}{s \rho P+1}\right), \\
& \mathcal{U}^{\prime \prime}\left(\mathbf{x}_{B}, s\right)=\mathbf{E}\left(\prod_{\mathbf{x} \in \widetilde{\Omega}\left(\mathbf{x}_{B}\right)} u^{\prime}\left(\mathbf{x}, \mathbf{x}_{B}, s\right)\right) \\
= & \exp \left(-\lambda \int_{\mathcal{B}\left(\mathbf{x}_{B}, R\right)} \frac{\frac{s P|\mathbf{x}-\mathcal{B S}(\mathbf{x})|^{\alpha}}{\left|\mathbf{x}-\mathbf{x}_{B}\right|^{\alpha}}}{\frac{s P|\mathbf{x}-\mathcal{B S}(\mathbf{x})|^{\alpha}}{\left|\mathbf{x}-\mathbf{x}_{B}\right|^{\alpha}}+1} d \mathbf{x}\right) .
\end{aligned}
$$

Then, following the same steps as $(9), \mathcal{L}_{I^{\prime}}\left(\mathbf{x}_{B}, s\right)$ is derived as

$$
\begin{aligned}
& \mathcal{L}_{I^{\prime}}\left(\mathbf{x}_{B}, s\right)=\mathcal{L}_{0}^{\prime}\left(\mathbf{x}_{B}, s\right) \exp \left(-\mu \int_{\mathbb{R}^{2}}(1-\right. \\
& \left.\left.\frac{\mathcal{V}^{\prime}\left(\mathbf{x}_{0}, \mathbf{x}_{B}, s\right) \mathcal{W}^{\prime}\left(\mathbf{x}_{0}, \mathbf{x}_{B}, s\right)}{\mathcal{U}^{\prime}\left(\mathbf{x}_{0}, \mathbf{x}_{B}, s\right)}\right) d \mathbf{x}_{0}\right) \frac{\mathcal{W}^{\prime \prime}\left(\mathbf{x}_{B}, s\right) \mathcal{V}^{\prime \prime}\left(\mathbf{x}_{B}, s\right)}{\mathcal{U}^{\prime \prime}\left(\mathbf{x}_{B}, s\right)} .
\end{aligned}
$$

An intuitive explanation to the above is as follows. First, $\mathcal{L}_{0}^{\prime}\left(\mathbf{x}_{B}, s\right)$ corresponds to the interference of all macrocell UEs. Second, similar to the discussions in Section IV-A3, $\frac{\mathcal{V}^{\prime}\left(\mathbf{x}_{0}, \mathbf{x}_{B}, s\right) \mathcal{W}^{\prime}\left(\mathbf{x}_{0}, \mathbf{x}_{B}, s\right)}{\mathcal{U}^{\prime}\left(\mathbf{x}_{0}, \mathbf{x}_{B}, s\right)}$ represents the overall interference variation when a femtocell centered at $\mathbf{x}_{0}$ is added. Third, $\exp \left(-\mu \int_{\mathbb{R}^{2}}\left(1-\frac{\mathcal{V}^{\prime}\left(\mathbf{x}_{0}, \mathbf{x}_{B}, s\right) \mathcal{W}^{\prime}\left(\mathbf{x}_{0}, \mathbf{x}_{B}, s\right)}{\mathcal{U}^{\prime}\left(\mathbf{x}_{0}, \mathbf{x}_{B}, s\right)}\right) d \mathbf{x}_{0}\right)$ is the overall interference variation after adding all femtocells other than the typical femtocell. Fourth, $\frac{\mathcal{W}^{\prime \prime}\left(\mathbf{x}_{B}, s\right) \mathcal{V}^{\prime \prime}\left(\mathbf{x}_{B}, s\right)}{\mathcal{U}^{\prime \prime}\left(\mathbf{x}_{B}, s\right)}$ represents the overall interference variation after adding the typical femtocell. As a consequence, the overall interference can be computed as formula (33).

4) Outage Probability: Similar to (11), the outage probability (given $\mathbf{x}_{B}$ ) is

$\widehat{P}_{\text {out }}^{o}\left(\mathbf{x}_{B}\right)=\mathbf{P}\left(Q h_{\mathbf{x}_{U}, \mathbf{x}_{B}}<T I^{\prime}\left(\mathbf{x}_{B}\right)\right)=1-\left.\mathcal{L}_{I^{\prime}}\left(\mathbf{x}_{B}, s\right)\right|_{s=T^{\prime}}$,

where $\mathbf{x}_{U}$ is the coordinate of the typical femtocell UE (irrelevant to the result), $T^{\prime}=\frac{T}{Q}$, and $T$ is the SIR threshold. Because $\mathbf{x}_{B}$ is uniformly distributed in $\mathcal{H}(\mathbf{0})$, the average outage probability can be computed as $\int_{\mathcal{H}(\mathbf{0})} \widehat{P}_{\text {out }}^{o}\left(\mathbf{x}_{B}\right) d \mathbf{x}_{B} /|\mathcal{H}(\mathbf{0})|$, where $|\mathcal{H}(\mathbf{0})|=\frac{3 \sqrt{3} R_{c}^{2}}{2}$ is the area of a macrocell.

\section{B. Closed Access Case}

The overall interference has three parts: from macrocell UEs $\left(\widehat{I}_{1}^{\prime}\left(\mathbf{x}_{B}\right)\right)$, from femtocell UEs outside the typical femtocell $\left(\widehat{I}_{3}^{\prime}\left(\mathbf{x}_{B}\right)\right)$, and from femtocell UEs inside the typical femtocell $\left(\widehat{I}_{4}^{\prime}\left(\mathbf{x}_{B}\right)\right) . \widehat{I}_{1}^{\prime}\left(\mathbf{x}_{B}\right)$ can be computed as

$$
\widehat{I}_{1}^{\prime}\left(\mathbf{x}_{B}\right)=\sum_{\mathbf{x} \in \Phi} \frac{P|\mathbf{x}-\mathcal{B S}(\mathbf{x})|^{\alpha} h_{\mathbf{x}, \mathbf{x}_{B}}}{\left|\mathbf{x}-\mathbf{x}_{B}\right|^{\alpha}}
$$

and $\widehat{I}_{3}^{\prime}\left(\mathbf{x}_{B}\right)$ and $\widehat{I}_{4}^{\prime}\left(\mathbf{x}_{B}\right)$ are exactly the same as $I_{3}^{\prime}\left(\mathbf{x}_{B}\right)$ in (22) and $I_{4}^{\prime}\left(\mathbf{x}_{B}\right)$ in (23), respectively.

The overall interference is $\widehat{I}^{\prime}\left(\mathbf{x}_{B}\right)=\widehat{I}_{1}^{\prime}\left(\mathbf{x}_{B}\right)+\widehat{I}_{3}^{\prime}\left(\mathbf{x}_{B}\right)+$ $\widehat{I}_{4}^{\prime}\left(\mathbf{x}_{B}\right)$. Then, the Laplace transform of $\widehat{I}^{\prime}\left(\mathbf{x}_{B}\right)$ is

$$
\begin{aligned}
& \mathcal{L}_{\widehat{I}^{\prime}}\left(\mathbf{x}_{B}, s\right)=\mathcal{L}_{0}^{\prime}\left(\mathbf{x}_{B}, s\right) \\
& \exp \left(-\mu \int_{\mathbb{R}^{2}}\left(1-\mathcal{W}^{\prime}\left(\mathbf{x}_{0}, \mathbf{x}_{B}\right)\right) d \mathbf{x}_{0}\right) \cdot \mathcal{W}^{\prime \prime}\left(\mathbf{x}_{B}, s\right)
\end{aligned}
$$

The outage probability (given $\mathbf{x}_{B}$ ) is

$$
\widehat{P}_{\text {out }}^{c}\left(\mathbf{x}_{B}\right)=1-\left.\mathcal{L}_{\widehat{I}^{\prime}}\left(\mathbf{x}_{B}, s\right)\right|_{s=T^{\prime}} .
$$

The average outage probability is $\int_{\mathcal{H}(\mathbf{0})} \widehat{P}_{\text {out }}^{c}\left(\mathbf{x}_{B}\right) d \mathbf{x}_{B} /|\mathcal{H}(\mathbf{0})|$. Similar to the discussion in Section IV-C, we still can normalize $R_{c}$ and $P$. Hence, in the rest of this section, without loss of generality, we set $R_{c}=1$ and $P=1$.

\section{Open Access vs. Closed Access}

In this subsection, we compare the outage performance of open access and closed access at the femtocell level.

$$
\text { Let } \mathbf{V}_{\max }^{\prime} \triangleq 4 \pi^{2} R^{4}\left(T^{\prime} \rho\right)^{\frac{2}{\alpha}}\left(\frac{1}{8}+\frac{1}{4(\alpha+2)}+\frac{1}{(\alpha+2)(\alpha-2)}\right) \text {, }
$$
$\mathbf{V}_{\text {min }}^{\prime} \triangleq 2 \pi^{2} R^{4}\left(T^{\prime} \rho\right)^{\frac{2}{\alpha}}\left(\frac{1}{8}+\frac{1}{4(\alpha+2)}+\frac{1}{(\alpha+2)(\alpha-2)}\right), C_{u}^{\prime}$ be a system-level constant shown in (74), $\mathcal{R}_{\min }$ and $\mathcal{R}_{\max }$ be as shown in (75) and (76) in the proof of Theorem 4, which are in closed forms if $\alpha$ is a rational number ${ }^{4}$. Then we have the following theorem:

Theorem 4. Given $\mathbf{x}_{B}$, a sufficient condition for $\widehat{P}_{\text {out }}^{o}\left(\mathbf{x}_{B}\right)<$ $\widehat{P}_{\text {out }}^{c}\left(\mathbf{x}_{B}\right)$ is

$$
K_{1} \triangleq-\mu \mathbf{V}_{\max }^{\prime}+\mu \pi R^{2} C_{u}^{\prime} e^{-\bar{\nu}}-\frac{\pi R^{2} T^{\prime} \rho}{T^{\prime} \rho+1}+\mathcal{R}_{\min }>0,
$$

and a sufficient condition for $\widehat{P}_{\text {out }}^{o}\left(\mathbf{x}_{B}\right)>\widehat{P}_{\text {out }}^{c}\left(\mathbf{x}_{B}\right)$ is

$K_{2} \triangleq-\mu \pi R^{2} C_{u}^{\prime} e^{\bar{\lambda}}+\mu \mathbf{V}_{\min }^{\prime} e^{-\bar{\nu}-\bar{\lambda}}+\frac{\pi R^{2} T^{\prime} \rho}{T^{\prime} \rho+1}-\mathcal{R}_{\max }>0$

Proof: See Appendix-D for the proof.

Through Theorem 4, the closed-form expressions can be used to compare the outage probabilities between open access and closed access without the computational complexity introduced by numeric integrations in (34) and (37).

Similar to the discussion in Section IV-D, let $\rho^{* *}$ denote the threshold value of $\rho$ such that $\widehat{P}_{\text {out }}^{o}\left(\mathbf{x}_{B}\right)=\widehat{P}_{\text {out }}^{c}\left(\mathbf{x}_{B}\right)$. At the femtocell level, given that a femtocell BS is located at $\mathbf{x}_{B}$ (the relative coordinate w.r.t. the nearest macrocell), its local femtocell UEs experience less outage iff $\rho<\rho^{* *}$. Thus, $\rho^{* *}$ is referred to as the maximum power enhancement tolerated by the femtocell.

Instead of deriving $\rho^{* *}$ through (34) and (37), which introduces high computational complexity due to multiple levels of integration, we can find the lower bound $\rho_{\text {min }}^{* *}$ and upper bound $\rho_{\max }^{* *}$ of $\rho^{* *}$ through Theorem 4 . Accordingly, $\rho_{\min }^{* *}$ is the value satisfying $K_{1}=0$ and $\rho_{\max }^{* *}$ is the value satisfying $K_{2}=0$. Thus, $\rho_{\min }^{* *}$ and $\rho_{\max }^{* *}$ can be found by a numerical search approach w.r.t. the closed-form expressions.

${ }^{4}$ It is acceptable to assume $\alpha$ as a rational number in reality, because each real number can be approximated by a rational number with arbitrary precision. 


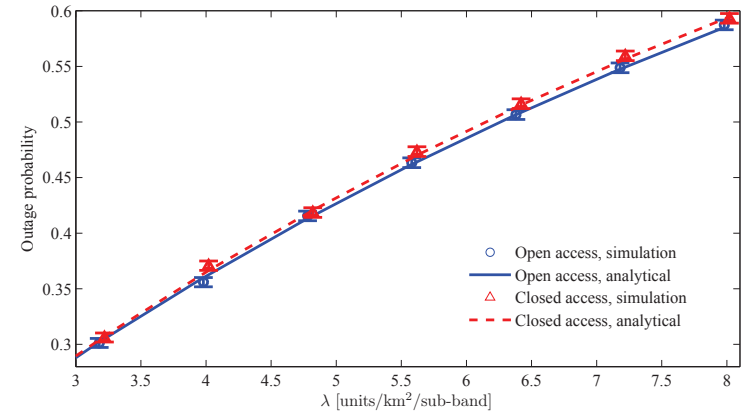

Fig. 2. Macrocell outage probability under different $\lambda$, with $\mu=4$ units $/ \mathrm{km}^{2} /$ sub-band and $T=0.1$.

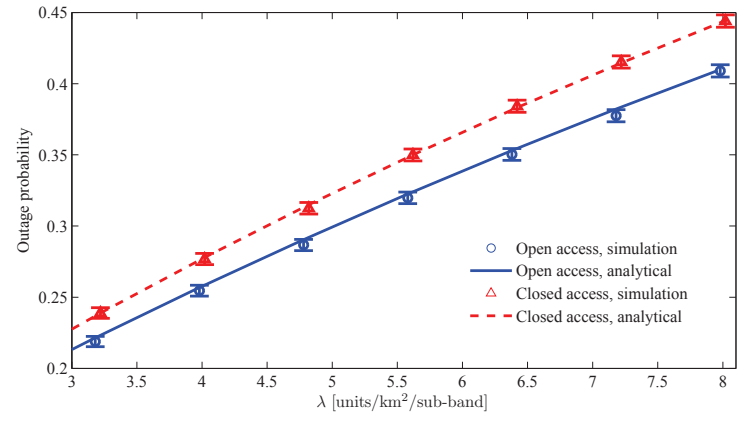

Fig. 3. Femtocell outage probability under different $\lambda$, with $\mu=4$ units $/ \mathrm{km}^{2} /$ sub-band and $T=0.1$.

\section{NUMERICAL STUdY}

We present simulation and numerical studies on the outage performance in the two-tier network with femtocells. First, we study the performance of open access and closed access under different user densities, femtocell densities, and SIR thresholds. Second, we present the numerical results of $\rho^{*}$ and $\rho^{* *}$. Unless otherwise stated, $R_{c}=500 \mathrm{~m}, R=50 \mathrm{~m}, \alpha=3$; and fast fading is Rayleigh with unit mean. Each simulation data point is averaged over 50000 trials.

First, we study the performance under different $\mu, \lambda$, and $T$. The network parameters are as follows: $\nu(\mathbf{x})=80$ units $/ \mathrm{km}^{2} /$ sub-band if $|\mathbf{x}|<R$, and $\nu(\mathbf{x})=0$ otherwise; $P=-60 \mathrm{dBm}$, and $Q=P^{\prime}=-54 \mathrm{dBm}(\rho=6 \mathrm{~dB})$. Figs. 2 and 3 show the uplink outage probabilities of macrocell and femtocell UEs under different $\lambda$; Figs. 4 and 5 show the same under different $\mu$; and Figs. 6 and 7 show the same under different $T$. The analytical results are derived from the exact expressions in Sections IV-A, IV-B, V-A, and $\mathrm{V}-\mathrm{B}$, without applying any bounds. The error bars show the $95 \%$ confidence intervals for simulation results. For easier inspection, in Figs. 2-7, the plot points are slightly shifted horizontally to avoid overlapping error bars. The figures illustrate the accuracy of our analytical results. In addition, the figures show that the macrocell UE density strongly influences the outage probability of both macrocell and femtocell UEs, while the femtocell density only has a slight influence. At the macrocell level, increasing the density of femtocell leads to more proportion of macrocell UEs becoming open access UEs, which gives higher performance gap between open access and closed access. At the femtocell level, the interference is observed at femtocell BSs, and the average number of

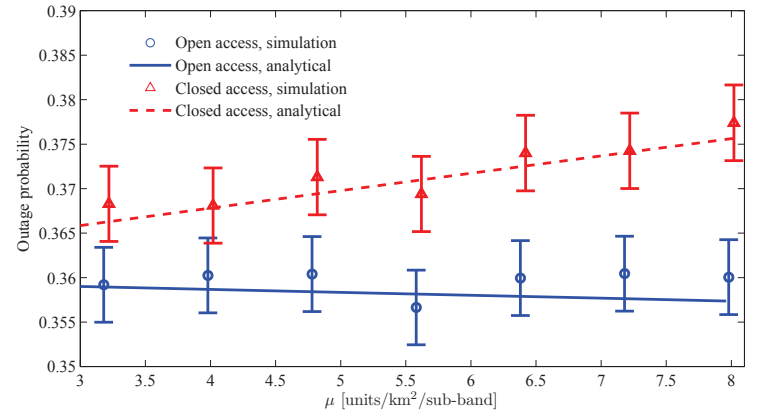

Fig. 4. Macrocell outage probability under different $\mu$, with $\lambda=4$ units $/ \mathrm{km}^{2} /$ sub-band and $T=0.1$.

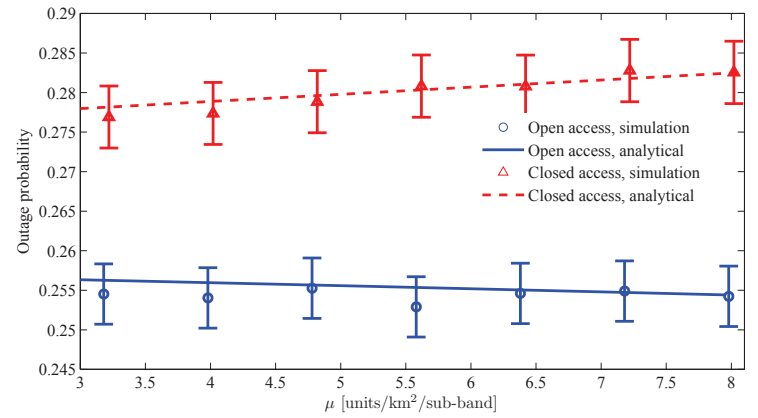

Fig. 5. Femtocell outage probability under different $\mu$, with $\lambda=4$ units $/ \mathrm{km}^{2} /$ sub-band and $T=0.1$.

macrocell UEs in a femtocell becomes a more important factor influencing the performance gap.

Next, we present the numerical results of $\rho^{*}$ and $\rho^{* *}$. The network parameters are as follows: $\lambda=4$ units $/ \mathrm{km}^{2} /$ sub-band; $\mu=4$ units $/ \mathrm{km}^{2} /$ sub-band; $\nu(\mathbf{x})=20$ units $/ \mathrm{km}^{2} /$ sub-band if $|\mathbf{x}|<R$, and $\nu(\mathbf{x})=0$ otherwise; $P=-60 \mathrm{dBm}$, and $Q=-54 \mathrm{dBm}$.

Fig. 8 presents the value of $\rho^{*}$ at the macrocell level. We compute the actual value of $\rho^{*}$ by numerically searching for the value such that (11) is equal to (14). Through the closedform expressions in Theorem 2, we are able to derive the upper and lower bounds of $\rho^{*}$. Through simulation, we can also search for the value of $\rho^{*}$ such that the simulated outage probability of open access is equal to that of closed access. Furthermore, we also simulate a more general scenario, where the received power is randomly distributed, rather than perfectly fixed to a single level. We study the scenario where the received power level of macrocell UEs is randomly distributed among $0.5 P, P, 1.5 P$, and $2 P$ with equal probability. If a macrocell UE is handed off to a femtocell, then its targeted received power is multiplied by $\rho$. The figure shows that $\rho^{*}$ is indeed within the upper bound and the lower bound, and the simulated $\rho^{*}$ agrees with the analytical $\rho^{*}$, validating the correctness of our analysis. Furthermore, this remains the case when the targeted received power is random, indicating the usefulness of our analysis in more practical scenarios.

Figs. 9, 10, and 11 present the value of $\rho^{* *}$ at the femtocell level. Fig. 9 shows $\rho^{* *}$ under different $R$ as we fixed $\mathbf{x}_{B}=$ $(0,100 \mathrm{~m})$; Figs. 10 and 11 show $\rho^{* *}$ under different $\mathbf{x}_{B}$ as we fixed $R=50 \mathrm{~m}$. The $y$-coordinates of $\mathbf{x}_{B}, y_{B}$, are fixed to 0 in Fig. 10, and the $x$-coordinates of $\mathbf{x}_{B}, x_{B}$, are fixed 


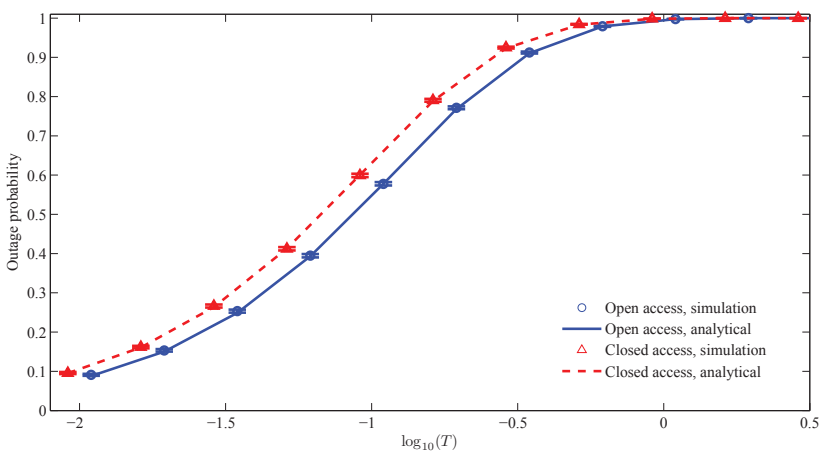

Fig. 6. Macrocell outage probability under different $T$, with $\lambda=8$ units $/ \mathrm{km}^{2} / \mathrm{sub}$-band and $\mu=8$ units $/ \mathrm{km}^{2} /$ sub-band.

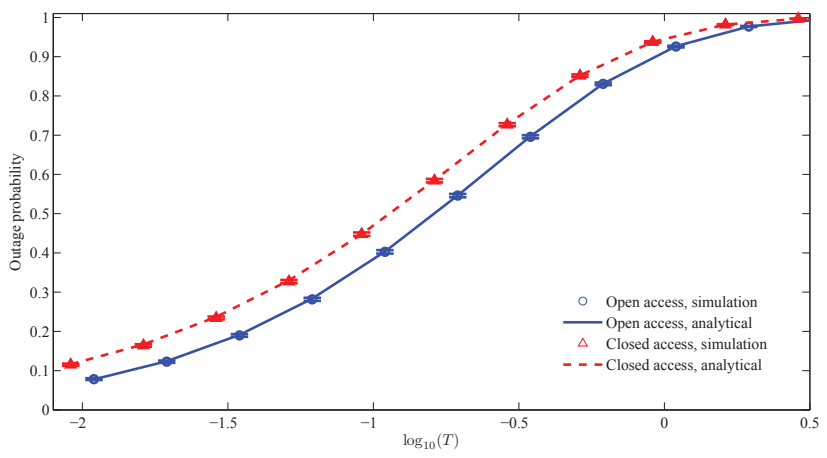

Fig. 7. Femtocell outage probability under different $T$, with $\lambda=8$ units $/ \mathrm{km}^{2} / \mathrm{sub}$-band and $\mu=8$ units $/ \mathrm{km}^{2} /$ sub-band.

to 0 in Fig. 11. The results show that $\rho^{* *}$ is indeed within the upper and lower bounds, and the simulated values of $\rho^{* *}$ agree with their analytical values, validating the correctness of our analysis. Furthermore, $\rho^{* *}$ decreases in $R$ at a rate slightly faster than that of $\rho^{*}$, while it increases in $x_{B}$ and $y_{B}$, until saturating when the femtocell BS is near the macrocell edge. This quantifies when femtocells are more beneficial as they decrease in size and increase in distance away from the macrocell BS.

\section{CONCLUSiOnS}

We have presented a theoretical framework to analyze the performance difference between open access and closed access in a two-tier femtocell network. Through establishing a stochastic geometric model, we capture the spatial patterns of different network components. We derive the numerical outage probabilities of open access and closed access at the macrocell and femtocell levels. As in most uplink interference analysis, the outage probability expressions are in non-closed forms. Hence, we further derive closed-form bounds for the maximum tolerated received power enhancement, to compare the two access modes. Simulations and numerical studies are conducted, validating the correctness of the analytical model as well as the usefulness of the bounds even when the received power is random.

In evaluating the performance difference between open access and closed access modes, so far we have used only the outage probability. In general, other performance metrics, such as data rate and handoff rate [36]-[38], may be of important

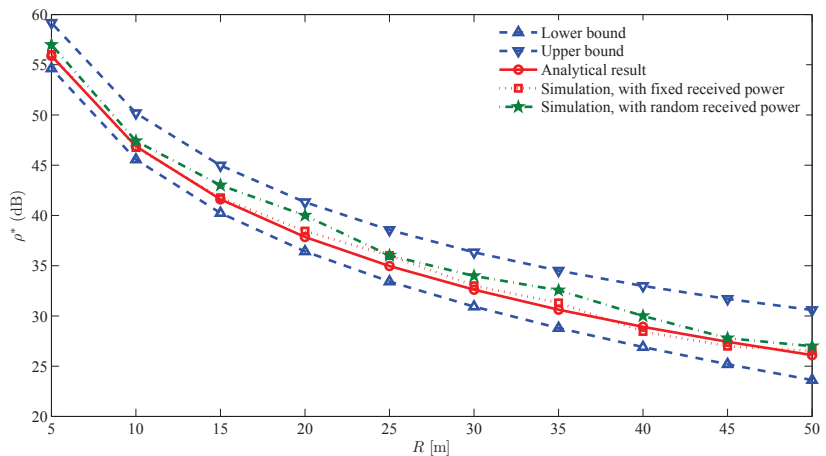

Fig. 8. $\rho^{*}$ under different $R$ at the macrocell level.

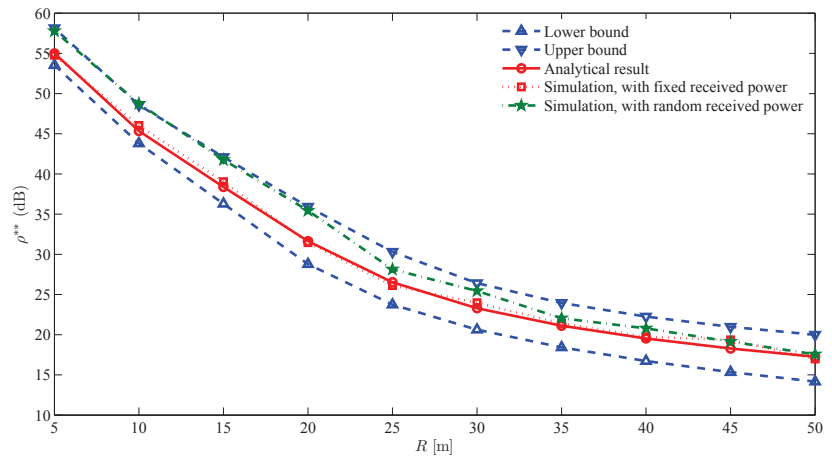

Fig. 9. $\quad \rho^{* *}$ under different $R$ at the femtocell level.

consideration. Other factors such as control overhead, system security, and regulatory policies further complicate the system design. A holistic comparison between the two access modes remains an open problem for future research.

\section{APPENDIX}

\section{A. Proof of Theorem 1}

Proof: The steps to derive Theorem 1 is shown in (40)(44) at the top of next page, where $\Phi^{0}$ is the point process corresponding to macrocell UEs not inside any femtocell, $\Phi^{1}$ is the point process corresponding to macrocell UEs inside some femtocell, and $\Phi$ is the aggregation of $\Phi^{0}$ and $\Phi^{1}$.

By the law of total expectation, we derive (41) from (40). $\Phi^{1}$ can be rewritten as the union of all the open access UEs in each femtocell, so $\mathbf{E}\left(\prod_{\mathbf{x} \in \Phi^{1}} u(\mathbf{x}, s) \mid \Theta\right)$ is equal to $\mathbf{E}\left(\prod_{\mathbf{x}_{0} \in \Theta} \prod_{\mathbf{x} \in \Omega\left(\mathbf{x}_{0}\right)} u(\mathbf{x}, s) \mid \Theta\right)$. In addition, because $\Phi$ is the aggregation of $\Phi^{0}$ and $\Phi^{1}, \mathbf{E}\left(\prod_{\mathbf{x} \in \Phi^{0}} u(\mathbf{x}, s) \mid \Theta\right)$ $\mathbf{E}\left(\prod_{\mathbf{x} \in \Phi^{1}} u(\mathbf{x}, s) \mid \Theta\right)$ is equal to $\mathbf{E}\left(\prod_{\mathbf{x} \in \Phi} u(\mathbf{x}, s) \mid \Theta\right)$. By considering the two equalities, we derive (43) from (42). Finally, we obtain (44) from the law of total expectation.

\section{B. Proof of Theorem 2}

Proof: We use the fact that $P$ and $R_{c}$ can be normalized and set $P=R_{c}=1$. Furthermore, we substitute $s=T$ into 


$$
\begin{aligned}
& \mathcal{L}_{I}(s)=\mathbf{E}(\exp (-s I))=\mathbf{E}\left[\prod_{\mathbf{x} \in \Phi^{0}} u(\mathbf{x}, s) \prod_{\mathbf{x}_{0} \in \Theta} \prod_{\mathbf{x} \in \Omega\left(\mathbf{x}_{0}\right)} v\left(\mathbf{x}, \mathbf{x}_{0}, s\right) \prod_{\mathbf{x}_{0} \in \Theta} \prod_{\mathbf{x} \in \Psi\left(\mathbf{x}_{0}\right)} w\left(\mathbf{x}, \mathbf{x}_{0}, s\right)\right] \\
& =\mathbf{E}\left[\mathbf{E}\left(\prod_{\mathbf{x} \in \Phi^{0}} u(\mathbf{x}, s) \mid \Theta\right) \mathbf{E}\left(\prod_{\mathbf{x}_{0} \in \Theta} \prod_{\mathbf{x} \in \Omega\left(\mathbf{x}_{0}\right)} v\left(\mathbf{x}, \mathbf{x}_{0}, s\right) \mid \Theta\right) \mathbf{E}\left(\prod_{\mathbf{x}_{0} \in \Theta} \prod_{\mathbf{x} \in \Psi\left(\mathbf{x}_{0}\right)} w\left(\mathbf{x}, \mathbf{x}_{0}, s\right) \mid \Theta\right)\right] \\
& =\mathbf{E}\left[\mathbf{E}\left(\prod_{\mathbf{x} \in \Phi^{0}} u(\mathbf{x}, s) \mid \Theta\right) \frac{\mathbf{E}\left(\prod_{\mathbf{x} \in \Phi^{1}} u(\mathbf{x}, s) \mid \Theta\right)}{\mathbf{E}\left(\prod_{\mathbf{x} \in \Phi^{1}} u(\mathbf{x}, s) \mid \Theta\right)} \mathbf{E}\left(\prod_{\mathbf{x}_{0} \in \Theta} \prod_{\mathbf{x} \in \Omega\left(\mathbf{x}_{0}\right)} v\left(\mathbf{x}, \mathbf{x}_{0}, s\right) \mid \Theta\right) \mathbf{E}\left(\prod_{\mathbf{x}_{0} \in \Theta} \prod_{\mathbf{x} \in \Psi\left(\mathbf{x}_{0}\right)} w\left(\mathbf{x}, \mathbf{x}_{0}, s\right) \mid \Theta\right)\right] \\
& =\mathbf{E}\left[\mathbf{E}\left(\prod_{\mathbf{x} \in \Phi} u(\mathbf{x}, s) \mid \Theta\right) \frac{\mathbf{E}\left(\prod_{\mathbf{x}_{0} \in \Theta} \prod_{\mathbf{x} \in \Omega\left(\mathbf{x}_{0}\right)} v\left(\mathbf{x}, \mathbf{x}_{0}, s\right) \mid \Theta\right)}{\mathbf{E}\left(\prod_{\mathbf{x}_{0} \in \Theta} \prod_{\mathbf{x} \in \Omega\left(\mathbf{x}_{0}\right)} u(\mathbf{x}, s) \mid \Theta\right)} \mathbf{E}\left(\prod_{\mathbf{x}_{0} \in \Theta} \prod_{\mathbf{x} \in \Psi\left(\mathbf{x}_{0}\right)} w\left(\mathbf{x}, \mathbf{x}_{0}, s\right) \mid \Theta\right)\right] \\
& =\mathbf{E}\left(\prod_{\mathbf{x} \in \Phi} u(\mathbf{x}, s)\right) \mathbf{E}\left[\prod_{\mathbf{x}_{0} \in \Theta}\left(\frac{\mathbf{E}\left(\prod_{\mathbf{x} \in \Omega\left(\mathbf{x}_{0}\right)} v\left(\mathbf{x}, \mathbf{x}_{0}, s\right)\right)}{\mathbf{E}\left(\prod_{\mathbf{x} \in \Omega\left(\mathbf{x}_{0}\right)} u(\mathbf{x}, s)\right)} \mathbf{E}\left(\prod_{\mathbf{x} \in \Psi\left(\mathbf{x}_{0}\right)} w\left(\mathbf{x}, \mathbf{x}_{0}, s\right)\right)\right)\right] .
\end{aligned}
$$

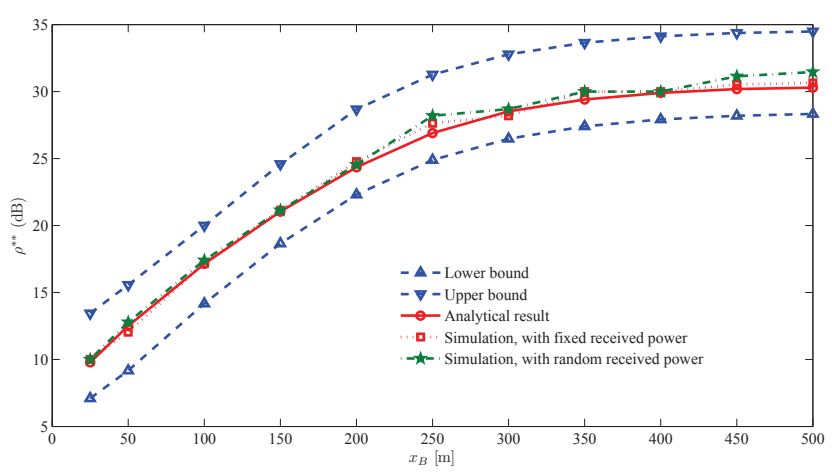

Fig. 10. $\rho^{* *}$ under different $\mathbf{x}_{B}, \mathbf{x}_{B}=\left(x_{B}, 0\right)$, at the femtocell level.

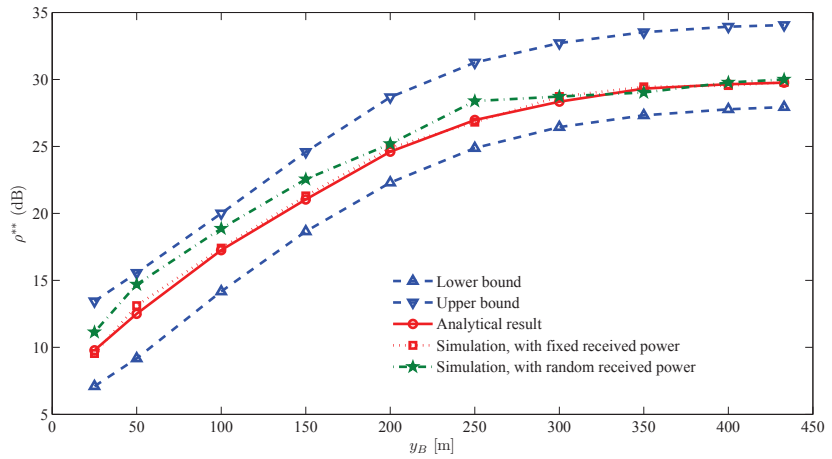

Fig. 11. $\rho^{* *}$ under different $\mathbf{x}_{B}, \mathbf{x}_{B}=\left(0, y_{B}\right)$, at the femtocell level.

(6), (7), and (8) such that

$$
\begin{aligned}
& \mathcal{W}\left(\mathbf{x}_{0}, T\right)=\exp \left(-\int_{\mathcal{B}(\mathbf{0}, R)} \frac{\frac{T Q|\mathbf{x}|^{\alpha}}{\left|\mathbf{x}+\mathbf{x}_{0}\right|^{\alpha}}}{\frac{T Q|\mathbf{x}|^{\alpha}}{\left|\mathbf{x}+\mathbf{x}_{0}\right|^{\alpha}}+1} \nu(\mathbf{x}) d \mathbf{x}\right), \\
& \mathcal{V}\left(\mathbf{x}_{0}, T\right)=\exp \left(-\lambda \int_{\mathcal{B}(\mathbf{0}, R)} \frac{\frac{T \rho|\mathbf{x}|^{\alpha}}{\left|\mathbf{x}+\mathbf{x}_{0}\right|^{\alpha}}}{\frac{T \rho|\mathbf{x}|^{\alpha}}{\left|\mathbf{x}+\mathbf{x}_{0}\right|^{\alpha}}+1} d \mathbf{x}\right),
\end{aligned}
$$

$$
\mathcal{U}\left(\mathbf{x}_{0}, T\right)=\exp \left(-\lambda \int_{\mathcal{B}\left(\mathbf{x}_{0}, R\right)} \frac{\frac{T|\mathbf{x}-\mathcal{B S}(\mathbf{x})|^{\alpha}}{|\mathbf{x}|^{\alpha}}}{\frac{T|\mathbf{x}-\mathcal{B S}(\mathbf{x})|^{\alpha}}{|\mathbf{x}|^{\alpha}}+1} d \mathbf{x}\right)
$$

(a) A sufficient condition for $P_{o u t}^{o}<P_{o u t}^{c}$

According to (10), (11), (13), and (14), $P_{\text {out }}^{o}<P_{\text {out }}^{c}$ iff

$$
\frac{\exp \left(-\mu \int_{\mathbb{R}^{2}}\left(1-\frac{\mathcal{V}\left(\mathbf{x}_{0}, T\right)}{\mathcal{U}\left(\mathbf{x}_{0}, T\right)} \mathcal{W}\left(\mathbf{x}_{0}, T\right)\right) d \mathbf{x}_{0}\right)}{\exp \left(-\mu \int_{\mathbb{R}^{2}}\left(1-\mathcal{W}\left(\mathbf{x}_{0}, T\right)\right) d \mathbf{x}_{0}\right)}>1,
$$

which is equivalent to

$$
\int_{\mathbb{R}^{2}}\left(\frac{\mathcal{V}\left(\mathbf{x}_{0}, T\right)}{\mathcal{U}\left(\mathbf{x}_{0}, T\right)}-1\right) \mathcal{W}\left(\mathbf{x}_{0}, T\right) d \mathbf{x}_{0}>0
$$

Let

$V\left(\mathbf{x}_{0}\right)=\int_{\mathcal{B}\left(\mathbf{x}_{0}, R\right)} \frac{\frac{T \rho\left|\mathbf{x}-\mathbf{x}_{0}\right|^{\alpha}}{|\mathbf{x}|^{\alpha}}}{\frac{T \rho\left|\mathbf{x}-\mathbf{x}_{0}\right|^{\alpha}}{|\mathbf{x}|^{\alpha}}+1} d \mathbf{x}=\int_{\mathcal{B}(\mathbf{0}, R)} \frac{\frac{T \rho|\mathbf{x}|^{\alpha}}{\left|\mathbf{x}+\mathbf{x}_{0}\right|^{\alpha}}}{\frac{T \rho|\mathbf{x}|^{\alpha}}{\left|\mathbf{x}+\mathbf{x}_{0}\right|^{\alpha}}+1} d \mathbf{x}$,

$U\left(\mathbf{x}_{0}\right)=\int_{\mathcal{B}\left(\mathbf{x}_{0}, R\right)} \frac{\frac{T|\mathbf{x}-\mathcal{B S}(\mathbf{x})|^{\alpha}}{|\mathbf{x}|^{\alpha}}}{\frac{T|\mathbf{x}-\mathcal{B S}(\mathbf{x})|^{\alpha}}{|\mathbf{x}|^{\alpha}}+1} d \mathbf{x}$.

Then, (49) becomes

$$
\int_{\mathbb{R}^{2}}\left(\frac{\exp \left(-\lambda V\left(\mathbf{x}_{0}\right)\right)}{\exp \left(-\lambda U\left(\mathbf{x}_{0}\right)\right)}-1\right) \mathcal{W}\left(\mathbf{x}_{0}, T\right) d \mathbf{x}_{0}>0 .
$$

Because $\exp (A)-1 \geq A$ for arbitrary $A$, and $\mathcal{W}\left(\mathbf{x}_{0}, T\right)>0$, we have $\int_{\mathbb{R}^{2}}\left(\frac{\exp \left(-\lambda V\left(\mathbf{x}_{0}\right)\right)}{\exp \left(-\lambda U\left(\mathbf{x}_{0}\right)\right)}-1\right) \mathcal{W}\left(\mathbf{x}_{0}, T\right) d \mathbf{x}_{0}>$ $\int_{\mathbb{R}^{2}}\left(-\lambda V\left(\mathbf{x}_{0}\right)+\lambda U\left(\mathbf{x}_{0}\right)\right) \mathcal{W}\left(\mathbf{x}_{0}, T\right) d \mathbf{x}_{0}$. Therefore, the following inequality is a sufficient condition for (52):

$$
\int_{\mathbb{R}^{2}}\left(-\lambda V\left(\mathbf{x}_{0}\right)+\lambda U\left(\mathbf{x}_{0}\right)\right) \mathcal{W}\left(\mathbf{x}_{0}, T\right) d \mathbf{x}_{0}>0 .
$$

Let $W_{\min }$ and $W_{\max }$ be the lower bound and upper bound of $\mathcal{W}\left(\mathbf{x}_{0}, T\right)$, respectively. According to (6), $W_{\max }=1$ and 
$W_{\min }=e^{-\bar{\nu}}$. Thus, the following is a sufficient condition for (53):

$$
-W_{\max } \int_{\mathbb{R}^{2}} V\left(\mathbf{x}_{0}\right) d \mathbf{x}_{0}+W_{\min } \int_{\mathbb{R}^{2}} U\left(\mathbf{x}_{0}\right) d \mathbf{x}_{0}>0 .
$$

Let $\mathbf{V}=\int_{\mathbb{R}^{2}} V\left(\mathbf{x}_{0}\right) d \mathbf{x}_{0}$, we have the following lemma corresponding to the upper and lower bounds of $\mathbf{V}$.

Lemma 1. Let

$$
\begin{aligned}
& \mathbf{V}_{\text {max }}=4 \pi^{2} R^{4}(T \rho)^{\frac{2}{\alpha}}\left(\frac{1}{8}+\frac{1}{4(\alpha+2)}+\frac{1}{(\alpha+2)(\alpha-2)}\right), \\
& \mathbf{V}_{\text {min }}=2 \pi^{2} R^{4}(T \rho)^{\frac{2}{\alpha}}\left(\frac{1}{8}+\frac{1}{4(\alpha+2)}+\frac{1}{(\alpha+2)(\alpha-2)}\right) .
\end{aligned}
$$

Then $\mathbf{V}_{\min } \leq \mathbf{V} \leq \mathbf{V}_{\max }$.

Proof: See Appendix-E for the proof.

Hence, the following is a sufficient condition for (54):

$$
-W_{\max } \mathbf{V}_{\max }+W_{\min } \int_{\mathbb{R}^{2}} U\left(\mathbf{x}_{0}\right) d \mathbf{x}_{0}>0 .
$$

In addition, we have

$$
\begin{aligned}
& \int_{\mathbb{R}^{2}} U\left(\mathbf{x}_{0}\right) d \mathbf{x}_{0}=\int_{\mathbb{R}^{2}} \int_{\mathcal{B}\left(\mathbf{x}_{0}, R\right)}\left(\frac{\frac{T|\mathbf{x}-\mathcal{B S}(\mathbf{x})|^{\alpha}}{|\mathbf{x}|^{\alpha}}}{\frac{T|\mathbf{x}-\mathcal{B S}(\mathbf{x})|^{\alpha}}{|\mathbf{x}|^{\alpha}}+1}\right) d \mathbf{x} d \mathbf{x}_{0} \\
& =\pi R^{2} \int_{\mathbb{R}^{2}}\left(\frac{\frac{T|\mathbf{x}-\mathcal{B S}(\mathbf{x})|^{\alpha}}{|\mathbf{x}|^{\alpha}}}{\frac{T|\mathbf{x}-\mathcal{B S}(\mathbf{x})|^{\alpha}}{|\mathbf{x}|^{\alpha}}+1}\right) d \mathbf{x}=\pi R^{2} C_{u}
\end{aligned}
$$

where

$$
C_{u}=\int_{\mathbb{R}^{2}}\left(\frac{\frac{T|\mathbf{x}-\mathcal{B S}(\mathbf{x})|^{\alpha}}{|\mathbf{x}|^{\alpha}}}{\frac{T|\mathbf{x}-\mathcal{B S}(\mathbf{x})|^{\alpha}}{|\mathbf{x}|^{\alpha}}+1}\right) d \mathbf{x}
$$

is only related to predetermined system-level constants $T$ and $\alpha$.

As a consequence, (57) becomes

$$
-W_{\max } \mathbf{V}_{\max }+W_{\min } \pi R^{2} C_{u}>0 .
$$

(b) A sufficient condition for $P_{\text {out }}^{o}>P_{\text {out }}^{c}$

According to (10), (11), (13), and (14), $P_{\text {out }}^{o}>P_{o u t}^{c}$ iff

$$
\frac{\exp \left(-\mu \int_{\mathbb{R}^{2}}\left(1-\mathcal{W}\left(\mathbf{x}_{0}, T\right)\right) d \mathbf{x}_{0}\right)}{\exp \left(-\mu \int_{\mathbb{R}^{2}}\left(1-\frac{\mathcal{V}\left(\mathbf{x}_{0}, T\right)}{\mathcal{U}\left(\mathbf{x}_{0}, T\right)} \mathcal{W}\left(\mathbf{x}_{0}, T\right)\right) d \mathbf{x}_{0}\right)}>1,
$$

Similar to the steps in (48)-(53), the following is a sufficient condition for (61):

$$
\int_{\mathbb{R}^{2}}\left(-\lambda U\left(\mathbf{x}_{0}, T\right)+\lambda V\left(\mathbf{x}_{0}, T\right)\right) \frac{\mathcal{V}\left(\mathbf{x}_{0}, T\right)}{\mathcal{U}\left(\mathbf{x}_{0}, T\right)} \mathcal{W}\left(\mathbf{x}_{0}, T\right) d \mathbf{x}_{0}>0 .
$$

Let $W_{\min }^{\prime}$ and $W_{\max }^{\prime}$ be the lower bound and upper bound of $\frac{\mathcal{V}\left(\mathbf{x}_{0}, T\right)}{\mathcal{U}\left(\mathbf{x}_{0}, T\right)} \mathcal{W}\left(\mathbf{x}_{0}, T\right)$, respectively. According to (6), (7), and (8), $W_{\max }^{\prime}=\exp (\bar{\lambda})$ and $W_{\min }^{\prime}=\exp (-\bar{\lambda}-\bar{\nu})$. Finally, the following is a sufficient condition for (62):

$$
-W_{\max }^{\prime} \pi R^{2} C_{u}+W_{\min }^{\prime} \mathbf{V}_{\min }>0
$$

\section{Proof of Theorem 3}

Proof: The steps to derive Theorem 3 is shown in (64)-(68) at the top of next page. Substituting (20)-(24) into (64), we derive (65). According to the law of total expectation, we derive (66) from (65). $\Phi^{1}$ can be rewritten as the union of all open access UEs in each femtocell (including the typical femtocell). Thus $\mathbf{E}\left(\prod_{\mathbf{x} \in \Phi^{1}} u^{\prime}\left(\mathbf{x}, \mathbf{x}_{B}, s\right) \mid \Theta\right)$ is equal to $\mathbf{E}\left(\prod_{\mathbf{x}_{0} \in \Theta} \prod_{\mathbf{x} \in \Omega\left(\mathbf{x}_{0}\right)} u^{\prime}\left(\mathbf{x}, \mathbf{x}_{B}, s\right) \mid \Theta\right)$. $\mathbf{E}\left(\prod_{\mathbf{x} \in \widetilde{\Omega}\left(\mathbf{x}_{B}\right)} u^{\prime}\left(\mathbf{x}, \mathbf{x}_{B}, s\right) \mid \Theta\right)$.

Also,

$\mathbf{E}\left(\prod_{\mathbf{x} \in \Phi^{0}} u^{\prime}\left(\mathbf{x}, \mathbf{x}_{B}, s\right) \mid \Theta\right) \cdot \mathbf{E}\left(\prod_{\mathbf{x} \in \Phi^{1}} u^{\prime}\left(\mathbf{x}, \mathbf{x}_{B}, s\right) \mid \Theta\right)$ is equal to $\mathbf{E}\left(\prod_{\mathbf{x} \in \Phi} u^{\prime}\left(\mathbf{x}, \mathbf{x}_{B}, s\right) \mid \Theta\right)$. By considering the two equalities, we derive (67) from (66). Finally, we derive (68) from the law of total expectation.

\section{Proof of Theorem 4}

Proof: We use the fact that $P$ and $R_{c}$ can be normalized and set $P=R_{c}=1$. Furthermore, we substitute $s=T^{\prime}$ into (27)-(32) such that

$$
\begin{aligned}
\mathcal{W}^{\prime}\left(\mathbf{x}_{0}, \mathbf{x}_{B}, T^{\prime}\right) & =\exp \left(-\int_{\mathcal{B}\left(\mathbf{x}_{0}, R\right)} \frac{\frac{T^{\prime} Q\left|\mathbf{x}-\mathbf{x}_{0}\right|^{\alpha}}{\left|\mathbf{x}-\mathbf{x}_{B}\right|^{\alpha}}}{\frac{T^{\prime} Q\left|\mathbf{x}-\mathbf{x}_{0}\right|^{\alpha}}{\left|\mathbf{x}-\mathbf{x}_{B}\right|^{\alpha}}+1} \nu\left(\mathbf{x}-\mathbf{x}_{0}\right) d \mathbf{x}\right), \\
\mathcal{V}^{\prime}\left(\mathbf{x}_{0}, \mathbf{x}_{B}, T^{\prime}\right) & =\exp \left(-\lambda \int_{\mathcal{B}\left(\mathbf{x}_{0}, R\right)} \frac{\frac{T^{\prime} \rho\left|\mathbf{x}-\mathbf{x}_{0}\right|^{\alpha}}{\left|\mathbf{x}-\mathbf{x}_{B}\right|^{\alpha}}}{\frac{T^{\prime} \rho\left|\mathbf{x}-\mathbf{x}_{0}\right|^{\alpha}}{\left|\mathbf{x}-\mathbf{x}_{B}\right|^{\alpha}}+1} d \mathbf{x}\right) \\
\mathcal{U}^{\prime}\left(\mathbf{x}_{0}, \mathbf{x}_{B}, T^{\prime}\right) & =\exp \left(-\lambda \int_{\mathcal{B}\left(\mathbf{x}_{0}, R\right)} \frac{\frac{T^{\prime}|\mathbf{x}-\mathcal{B S}(\mathbf{x})|^{\alpha}}{\left|\mathbf{x}-\mathbf{x}_{B}\right|^{\alpha}}}{\frac{T^{\prime}|\mathbf{x}-\mathcal{B S}(\mathbf{x})|^{\alpha}}{\left|\mathbf{x}-\mathbf{x}_{B}\right|^{\alpha}}+1} d \mathbf{x}\right) \\
\mathcal{V}^{\prime \prime}\left(\mathbf{x}_{B}, T^{\prime}\right) & \left.=e^{-\frac{T^{\prime} \rho \bar{\lambda}}{T^{\prime} \rho+1}}, \int_{\mathcal{B}\left(\mathbf{x}_{B}, R\right)} \frac{\frac{T^{\prime}|\mathbf{x}-\mathcal{B S}(\mathbf{x})|^{\alpha}}{\left|\mathbf{x}-\mathbf{x}_{B}\right|^{\alpha}}}{\frac{T^{\prime} \mid \mathbf{x}-\mathcal{B S}\left(\left.\mathbf{x}\right|^{\alpha}\right.}{\left|\mathbf{x}-\mathbf{x}_{B}\right|^{\alpha}}+1} d \mathbf{x}\right) \\
\mathcal{U}^{\prime \prime}\left(\mathbf{x}_{B}, T^{\prime}\right) & =\exp \left(-\lambda \int_{-},\right.
\end{aligned}
$$

(a) A sufficient condition for $\widehat{P}_{o u t}^{o}\left(\mathbf{x}_{B}\right)<\widehat{P}_{o u t}^{c}\left(\mathbf{x}_{B}\right)$ According to (33), (34), (36), and (37), $\widehat{P}_{\text {out }}^{o}\left(\mathbf{x}_{B}\right)<$ $\widehat{P}_{\text {out }}^{c}\left(\mathbf{x}_{B}\right)$ iff

$\frac{\exp \left(-\mu \int_{\mathbb{R}^{2}}\left(1-\frac{\mathcal{V}^{\prime}\left(\mathbf{x}_{0}, \mathbf{x}_{B}, T^{\prime}\right)}{\mathcal{U}^{\prime}\left(\mathbf{x}_{0}, \mathbf{x}_{B}, T^{\prime}\right)} \mathcal{W}^{\prime}\left(\mathbf{x}_{0}, \mathbf{x}_{B}, T^{\prime}\right)\right) d \mathbf{x}_{0}\right)}{\exp \left(-\mu \int_{\mathbb{R}^{2}}\left(1-\mathcal{W}^{\prime}\left(\mathbf{x}_{0}, \mathbf{x}_{B}, T^{\prime}\right)\right) d \mathbf{x}_{0}\right)} \frac{\mathcal{V}^{\prime \prime}\left(\mathbf{x}_{B}, T^{\prime}\right)}{\mathcal{U}^{\prime \prime}\left(\mathbf{x}_{B}, T^{\prime}\right)}>1$.

Let

$$
\begin{aligned}
V^{\prime}\left(\mathbf{x}_{0}, \mathbf{x}_{B}\right) & =\int_{\mathcal{B}\left(\mathbf{x}_{0}, R\right)} \frac{\frac{T^{\prime} \rho\left|\mathbf{x}-\mathbf{x}_{0}\right|^{\alpha}}{\left|\mathbf{x}-\mathbf{x}_{B}\right|^{\alpha}}}{\frac{T^{\prime} \rho\left|\mathbf{x}-\mathbf{x}_{0}\right|^{\alpha}}{\left|\mathbf{x}-\mathbf{x}_{B}\right|^{\alpha}}+1} d \mathbf{x} \\
U^{\prime}\left(\mathbf{x}_{0}, \mathbf{x}_{B}\right) & =\int_{\mathcal{B}\left(\mathbf{x}_{0}, R\right)}\left(\frac{\frac{T^{\prime}|\mathbf{x}-\mathcal{B S}(\mathbf{x})|^{\alpha}}{\left|\mathbf{x}-\mathbf{x}_{B}\right|^{\alpha}}}{\frac{T^{\prime}|\mathbf{x}-\mathcal{B S}(\mathbf{x})|^{\alpha}}{\left|\mathbf{x}-\mathbf{x}_{B}\right|^{\alpha}}+1}\right) d \mathbf{x} \\
\mathcal{R} & =\int_{\mathcal{B}\left(\mathbf{x}_{B}, R\right)} \frac{\frac{T^{\prime}|\mathbf{x}-\mathcal{B S}(\mathbf{x})|^{\alpha}}{\left|\mathbf{x}-\mathbf{x}_{B}\right|^{\alpha}}}{\frac{T^{\prime}|\mathbf{x}-\mathcal{B S}(\mathbf{x})|^{\alpha}}{\left|\mathbf{x}-\mathbf{x}_{B}\right|^{\alpha}}+1} d \mathbf{x} .
\end{aligned}
$$




$$
\begin{aligned}
& \mathbf{E}\left(\exp \left(-s\left(I^{\prime}\left(\mathbf{x}_{B}\right)\right)\right)\right) \\
& =\mathbf{E}\left[\prod_{\mathbf{x} \in \Phi^{0}} u^{\prime}\left(\mathbf{x}, \mathbf{x}_{B}, s\right) \cdot \prod_{\mathbf{x}_{0} \in \Theta} \prod_{\mathbf{x} \in \Omega\left(\mathbf{x}_{0}\right)} v^{\prime}\left(\mathbf{x}, \mathbf{x}_{0}, \mathbf{x}_{B}, s\right) \cdot \prod_{\mathbf{x}_{0} \in \Theta} \prod_{\mathbf{x} \in \Psi\left(\mathbf{x}_{0}\right)} w^{\prime}\left(\mathbf{x}, \mathbf{x}_{0}, \mathbf{x}_{B}, s\right) \cdot \prod_{\mathbf{x} \in \widetilde{\Omega}\left(\mathbf{x}_{B}\right)} v^{\prime}\left(\mathbf{x}, \mathbf{x}_{B}, \mathbf{x}_{B}, s\right) \prod_{\mathbf{x} \in \widetilde{\Psi}\left(\mathbf{x}_{B}\right)} w^{\prime}\left(\mathbf{x}, \mathbf{x}_{B}, \mathbf{x}_{B}, s\right)\right] \\
& =\mathbf{E}\left[\mathbf{E}\left(\prod_{\mathbf{x} \in \Phi^{0}} u^{\prime}\left(\mathbf{x}, \mathbf{x}_{B}, s\right) \mid \Theta\right) \frac{\mathbf{E}\left(\prod_{\mathbf{x} \in \Phi^{1}} u^{\prime}\left(\mathbf{x}, \mathbf{x}_{B}, s\right) \mid \Theta\right)}{\mathbf{E}\left(\prod_{\mathbf{x} \in \Phi^{1}} u^{\prime}\left(\mathbf{x}, \mathbf{x}_{B}, s\right) \mid \Theta\right)} \mathbf{E}\left(\prod_{\mathbf{x}_{0} \in \Theta} \prod_{\mathbf{x} \in \Omega\left(\mathbf{x}_{0}\right)} v^{\prime}\left(\mathbf{x}, \mathbf{x}_{0}, \mathbf{x}_{B}, s\right) \mid \Theta\right)\right. \\
& \left.\mathbf{E}\left(\prod_{\mathbf{x}_{0} \in \Theta} \prod_{\mathbf{x} \in \Psi\left(\mathbf{x}_{0}\right)} w^{\prime}\left(\mathbf{x}, \mathbf{x}_{0}, \mathbf{x}_{B}, s\right) \mid \Theta\right) \mathbf{E}\left(\prod_{\mathbf{x} \in \widetilde{\Omega}\left(\mathbf{x}_{B}\right)} v^{\prime}\left(\mathbf{x}, \mathbf{x}_{B}, \mathbf{x}_{B}, s\right) \mid \Theta\right) \mathbf{E}\left(\prod_{\mathbf{x} \in \widetilde{\Psi}\left(\mathbf{x}_{B}\right)} w^{\prime}\left(\mathbf{x}, \mathbf{x}_{B}, \mathbf{x}_{B}, s\right) \mid \Theta\right)\right] \\
& =\mathbf{E}\left[\mathbf{E}\left(\prod_{\mathbf{x} \in \Phi} u^{\prime}\left(\mathbf{x}, \mathbf{x}_{B}, s\right) \mid \Theta\right) \frac{\mathbf{E}\left(\prod_{\mathbf{x}_{0} \in \Theta} \prod_{\mathbf{x} \in \Omega\left(\mathbf{x}_{0}\right)} v^{\prime}\left(\mathbf{x}, \mathbf{x}_{0}, \mathbf{x}_{B}, s\right) \mid \Theta\right)}{\mathbf{E}\left(\prod_{\mathbf{x}_{0} \in \Theta} \prod_{\mathbf{x} \in \Omega\left(\mathbf{x}_{0}\right)} u^{\prime}\left(\mathbf{x}, \mathbf{x}_{B}, s\right) \mid \Theta\right)} \frac{\mathbf{E}\left(\prod_{\mathbf{x} \in \widetilde{\Omega}\left(\mathbf{x}_{B}\right)} v^{\prime}\left(\mathbf{x}, \mathbf{x}_{B}, \mathbf{x}_{B}, s\right) \mid \Theta\right)}{\mathbf{E}\left(\prod_{\mathbf{x} \in \widetilde{\Omega}\left(\mathbf{x}_{B}\right)} u^{\prime}\left(\mathbf{x}, \mathbf{x}_{B}, s\right) \mid \Theta\right)}\right. \\
& \left.\mathbf{E}\left(\prod_{\mathbf{x}_{0} \in \Theta} \prod_{\mathbf{x} \in \Psi\left(\mathbf{x}_{0}\right)} w^{\prime}\left(\mathbf{x}, \mathbf{x}_{0}, \mathbf{x}_{B}, s\right) \mid \Theta\right) \mathbf{E}\left(\prod_{\mathbf{x} \in \widetilde{\Psi}\left(\mathbf{x}_{B}\right)} w^{\prime}\left(\mathbf{x}, \mathbf{x}_{B}, \mathbf{x}_{B}, s\right) \mid \Theta\right)\right] \\
& =\mathbf{E}\left(\prod_{\mathbf{x} \in \Phi} u^{\prime}\left(\mathbf{x}, \mathbf{x}_{B}, s\right)\right) \mathbf{E}\left[\prod_{\mathbf{x}_{0} \in \Theta}\left(\frac{\mathbf{E}\left(\prod_{\mathbf{x} \in \Omega\left(\mathbf{x}_{0}\right)} v^{\prime}\left(\mathbf{x}, \mathbf{x}_{0}, \mathbf{x}_{B}, s\right)\right)}{\mathbf{E}\left(\prod_{\mathbf{x} \in \Omega\left(\mathbf{x}_{0}\right)} u^{\prime}\left(\mathbf{x}, \mathbf{x}_{B}, s\right)\right)} \mathbf{E}\left(\prod_{\mathbf{x} \in \Psi\left(\mathbf{x}_{0}\right)} w^{\prime}\left(\mathbf{x}, \mathbf{x}_{0}, \mathbf{x}_{B}, s\right)\right)\right)\right] \\
& \mathbf{E}\left(\prod_{\mathbf{x} \in \widetilde{\Psi}\left(\mathbf{x}_{B}\right)} w^{\prime}\left(\mathbf{x}, \mathbf{x}_{B}, \mathbf{x}_{B}, s\right)\right) \frac{\mathbf{E}\left(\prod_{\mathbf{x} \in \widetilde{\Omega}\left(\mathbf{x}_{B}\right)} v^{\prime}\left(\mathbf{x}, \mathbf{x}_{B}, \mathbf{x}_{B}, s\right)\right)}{\mathbf{E}\left(\prod_{\mathbf{x} \in \widetilde{\Omega}\left(\mathbf{x}_{B}\right)} u^{\prime}\left(\mathbf{x}, \mathbf{x}_{B}, s\right)\right)}
\end{aligned}
$$

Similar to (53), the following is a sufficient condition for (70):

$$
\begin{aligned}
& \mu \int_{\mathbb{R}^{2}}\left(-\lambda V^{\prime}\left(\mathbf{x}_{0}, \mathbf{x}_{B}\right)+\lambda U^{\prime}\left(\mathbf{x}_{0}, \mathbf{x}_{B}\right)\right) \mathcal{W}^{\prime}\left(\mathbf{x}_{0}, \mathbf{x}_{B}, T^{\prime}\right) d \mathbf{x}_{0} \\
& -\frac{\lambda \pi R^{2} T^{\prime} \rho}{T^{\prime} \rho+1}+\lambda \mathcal{R}>0 .
\end{aligned}
$$

Let $W_{\min }^{\prime \prime}$ and $W_{\max }^{\prime \prime}$ be the lower bound and upper bound of $\mathcal{W}^{\prime}\left(\mathbf{x}_{0}, \mathbf{x}_{B}, T^{\prime}\right)$, respectively. According to (27), $W_{\max }^{\prime \prime}=1$ and $W_{\min }^{\prime \prime}=\exp (-\bar{\nu})$. Thus, the following is a sufficient condition for (72):

$$
\begin{aligned}
& \mu \int_{\mathbb{R}^{2}}\left(-V^{\prime}\left(\mathbf{x}_{0}, \mathbf{x}_{B}\right) W_{\max }^{\prime \prime}+U^{\prime}\left(\mathbf{x}_{0}, \mathbf{x}_{B}\right) W_{\min }^{\prime \prime}\right) d \mathbf{x}_{0} \\
& -\frac{\pi R^{2} T^{\prime} \rho}{T^{\prime} \rho+1}+\mathcal{R}>0
\end{aligned}
$$

where $\int_{\mathbb{R}^{2}} V^{\prime}\left(\mathbf{x}_{0}, \mathbf{x}_{B}\right) d \mathbf{x}_{0}=\int_{\mathbb{R}^{2}} \int_{\mathcal{B}\left(\mathbf{x}_{0}, R\right)} \frac{\frac{T^{\prime} \rho\left|\mathbf{x}-\mathbf{x}_{0}\right|^{\alpha}}{|\mathbf{x}|^{\alpha}}}{\frac{T^{\prime} \rho\left|\mathbf{x}-\mathbf{x}_{0}\right|^{\alpha}}{|\mathbf{x}| \alpha}+1} d \mathbf{x} d \mathbf{x}_{0}$ is in the same form as (80). Thus, by applying Lemma 1, we can derive its upper bound and lower bound as $\mathbf{V}_{\max }^{\prime}$ and $\mathbf{V}_{\min }^{\prime}$ from (83) and (86), respectively. Similar to the derivation of (58), $\int_{\mathbb{R}^{2}} U^{\prime}\left(\mathbf{x}_{0}, \mathbf{x}_{B}\right) d \mathbf{x}_{0}=\pi R^{2} C_{u}^{\prime}$ where

$$
C_{u}^{\prime} \triangleq \int_{\mathbb{R}^{2}}\left(\frac{\frac{T^{\prime}|\mathbf{x}-\mathcal{B S}(\mathbf{x})|^{\alpha}}{\left|\mathbf{x}-\mathbf{x}_{B}\right|^{\alpha}}}{\frac{T^{\prime}|\mathbf{x}-\mathcal{B S}(\mathbf{x})|^{\alpha}}{\left|\mathbf{x}-\mathbf{x}_{B}\right|^{\alpha}}+1}\right) d \mathbf{x}
$$

In addition, the lower bound $\mathcal{R}_{\min }$ and the upper bound $\mathcal{R}_{\max }$ of $\mathcal{R}$ can be derived as follows:

$\mathcal{R}_{\text {min }}=$

$$
\begin{cases}\pi \int_{0}^{R} \frac{\frac{T^{\prime}\left(\left|\mathbf{x}_{B}\right|\right)^{\alpha}}{r^{\alpha}} r}{T^{\prime}\left(\left|\mathbf{x}_{B}\right|\right)^{\alpha}}+1 & \text { if }\left|\mathbf{x}_{B}\right| \leq R \\ \pi \int_{0}^{R} \frac{\frac{T^{\prime}\left(\left|\mathbf{x}_{B}\right|-R\right)^{\alpha}}{r^{\alpha}} r}{\frac{T^{\prime}\left(\left|\mathbf{x}_{B}\right|-R\right)^{\alpha}}{r^{\alpha}}+1} d r+\pi \int_{0}^{R} \frac{\frac{T^{\prime}\left(\left|\mathbf{x}_{B}\right|\right)^{\alpha}}{r^{\alpha}} r}{\frac{T^{\prime}\left(\left|\mathbf{x}_{B}\right|\right)^{\alpha}}{r^{\alpha}}+1} d r & \text { if }\left|\mathbf{x}_{B}\right|>R\end{cases}
$$

and

$$
\begin{aligned}
\mathcal{R}_{\max }=\pi \int_{0}^{R} \frac{\frac{T^{\prime}\left(\left|\mathbf{x}_{B}\right|+R\right)^{\alpha}}{r^{\alpha}} r}{\frac{T^{\prime}\left(\left|\mathbf{x}_{B}\right|+R\right)^{\alpha}}{r^{\alpha}}+1} d r+ \\
\pi \int_{0}^{R} \frac{\frac{T^{\prime}\left(\sqrt{\left|\mathbf{x}_{B}\right|^{2}+R^{2}}\right)^{\alpha}}{r^{\alpha}} r}{\frac{T^{\prime}\left(\sqrt{\left|\mathbf{x}_{B}\right|^{2}+R^{2}}\right)^{\alpha}}{r^{\alpha}}+1} d r .
\end{aligned}
$$

Note that $\int \frac{B r}{r^{\alpha}+B} d r$ is in closed form when $\alpha$ is a rational number. Therefore, both $\mathcal{R}_{\min }$ and $\mathcal{R}_{\max }$ are expressed in closed forms.

Finally, the following is a sufficient condition for (73):

$$
-\mu \mathbf{V}_{\max }^{\prime}+\mu \pi R^{2} C_{u}^{\prime} W_{\min }^{\prime \prime}-\frac{\pi R^{2} T^{\prime} \rho}{T^{\prime} \rho+1}+\mathcal{R}_{\min }>0
$$

(b) A sufficient condition for $\widehat{P}_{\text {out }}^{o}\left(\mathbf{x}_{B}\right)>\widehat{P}_{\text {out }}^{c}\left(\mathbf{x}_{B}\right)$

Similar to the derivations of (70) and (72), $\widehat{P}_{\text {out }}^{o}\left(\mathbf{x}_{B}\right)>$ $\widehat{P}_{\text {out }}^{c}\left(\mathbf{x}_{B}\right)$ iff

$\mu \int_{\mathbb{R}^{2}}\left(-\lambda U^{\prime}\left(\mathbf{x}_{0}, \mathbf{x}_{B}\right)+\lambda V^{\prime}\left(\mathbf{x}_{0}, \mathbf{x}_{B}\right)\right) \frac{\mathcal{W}^{\prime}\left(\mathbf{x}_{0}, \mathbf{x}_{B}, T^{\prime}\right) \mathcal{V}^{\prime}\left(\mathbf{x}_{0}, \mathbf{x}_{B}, T^{\prime}\right)}{\mathcal{U}^{\prime}\left(\mathbf{x}_{0}, \mathbf{x}_{B}, T^{\prime}\right)} d \mathbf{x}_{0}$
$+\frac{\lambda \pi R^{2} T^{\prime} \rho}{T^{\prime} \rho+1}-\lambda \mathcal{R}>0$.

Let $W_{\min }^{\prime \prime \prime}$ and $W_{\max }^{\prime \prime \prime}$ be the lower bound and upper bound value of $\frac{\mathcal{W}^{\prime}\left(\mathbf{x}_{0}, \mathbf{x}_{B}, T^{\prime}\right) \mathcal{V}^{\prime}\left(\mathbf{x}_{0}, \mathbf{x}_{B}, T^{\prime}\right)}{\mathcal{U}^{\prime}\left(\mathbf{x}_{0}, \mathbf{x}_{B}, T^{\prime}\right)}$, respectively. According to 
(27)-(29), $W_{\max }^{\prime \prime \prime}=\exp (\bar{\lambda})$ and $W_{\min }^{\prime \prime \prime}=\exp (-\bar{\lambda}-\bar{\nu})$. Then similar to the derivation of (77), we see that the following is a sufficient condition for (78):

$$
-\mu \pi R^{2} C_{u}^{\prime} W_{\max }^{\prime \prime \prime}+\mu \mathbf{V}_{\min }^{\prime} W_{\min }^{\prime \prime \prime}+\frac{\pi R^{2} T^{\prime} \rho}{T^{\prime} \rho+1}-\mathcal{R}_{\max }>0 .
$$

\section{E. Proof of Lemma 1}

\section{Proof: Upper Bound of V}

$$
\begin{aligned}
\mathbf{V}= & \int_{\mathbb{R}^{2}} \int_{\mathcal{B}\left(\mathbf{x}_{0}, R\right)} \frac{\frac{T \rho\left|\mathbf{x}-\mathbf{x}_{0}\right|^{\alpha}}{|\mathbf{x}|^{\alpha}}}{\frac{T \rho\left|\mathbf{x}-\mathbf{x}_{0}\right|^{\alpha}}{|\mathbf{x}|^{\alpha}}+1} d \mathbf{x} d \mathbf{x}_{0} \\
= & \int_{\mathbb{R}^{2}} \int_{\mathcal{B}(\mathbf{x}, R)} \frac{\frac{T \rho\left|\mathbf{x}-\mathbf{x}_{0}\right|^{\alpha}}{|\mathbf{x}|^{\alpha}}}{\frac{T \rho\left|\mathbf{x}-\mathbf{x}_{0}\right|^{\alpha}}{|\mathbf{x}|^{\alpha}}+1} d \mathbf{x}_{0} d \mathbf{x} \\
= & \int_{0}^{\infty} 2 \pi r_{1} \int_{0}^{R} \frac{\frac{T \rho r_{2}^{\alpha}}{r_{1}^{\alpha}}}{\frac{T \rho r_{2}^{\alpha}}{r_{1}^{\alpha}}+1} 2 \pi r_{2} d r_{2} d r_{1} \\
\leq & \int_{0}^{\infty} 2 \pi r_{1} \int_{0}^{R} \mathbf{1}\left(T \rho \frac{r_{2}^{\alpha}}{r_{1}^{\alpha}} \geq 1\right) 2 \pi r_{2} d r_{2} d r_{1}+ \\
& \int_{0}^{\infty} 2 \pi r_{1} \int_{0}^{R} \mathbf{1}\left(T \rho \frac{r_{2}^{\alpha}}{r_{1}^{\alpha}}<1\right) \frac{T \rho r_{2}^{\alpha}}{r_{1}^{\alpha}} 2 \pi r_{2} d r_{2} d r_{1} \\
= & 4 \pi^{2} R^{4}(T \rho)^{\frac{2}{\alpha}}\left(\frac{1}{8}+\frac{1}{4(\alpha+2)}+\frac{1}{(\alpha+2)(\alpha-2)}\right)
\end{aligned}
$$

In (81), the integrated item is in the form of $\frac{X}{X+1}$, where $X=\frac{T \rho r_{2}^{\alpha}}{r_{1}^{\alpha}} \geq 0$. The bound of the integrated item can be found as follows: if $X \geq 1, \frac{1}{2} \leq \frac{X}{X+1} \leq 1$; otherwise, if $X<1, \frac{X}{2} \leq \frac{X}{X+1} \leq X$. Accordingly, we can separate the integration region into the $\frac{T \rho r_{2}^{\alpha}}{r_{1}^{\alpha}} \geq 1$ region and the $\frac{T \rho r_{2}^{\alpha}}{r_{1}^{\alpha}}<1$ region. As a consequence, the upper bound of (81) can be derived as (82).

\section{Lower Bound of $\mathrm{V}$}

Following a similar approach as above, we have

$$
\begin{aligned}
\mathbf{V}= & \int_{0}^{\infty} 2 \pi r_{1} \int_{0}^{R} \frac{\frac{T \rho r_{2}^{\alpha}}{r_{1}^{\alpha}}}{\frac{T \rho r_{2}^{\alpha}}{r_{1}^{\alpha}}+1} 2 \pi r_{2} d r_{2} d r_{1} \\
\geq & \int_{0}^{\infty} 2 \pi r_{1} \int_{0}^{R} \mathbf{1}\left(T \rho \frac{r_{2}^{\alpha}}{r_{1}^{\alpha}} \geq 1\right) \pi r_{2} d r_{2} d r_{1}+ \\
& \int_{0}^{\infty} 2 \pi r_{1} \int_{0}^{R} \mathbf{1}\left(T \rho \frac{r_{2}^{\alpha}}{r_{1}^{\alpha}}<1\right) \frac{T \rho r_{2}^{\alpha}}{r_{1}^{\alpha}} \pi r_{2} d r_{2} d r_{1} \\
= & 2 \pi^{2} R^{4}(T \rho)^{\frac{2}{\alpha}}\left(\frac{1}{8}+\frac{1}{4(\alpha+2)}+\frac{1}{(\alpha+2)(\alpha-2)}\right)
\end{aligned}
$$

\section{REFERENCES}

[1] D. Knisely, T. Yoshizawa, and F. Favichia, "Standardization of femtocells in 3GPP," IEEE Commun. Mag., vol. 47, no. 9, pp. 68-75, Sep. 2009.

[2] H. Dhillon, R. Ganti, F. Baccelli, and J. Andrews, "A tractable framework for coverage and outage in heterogeneous cellular networks," in Inform. Theory and Applicat. Workshop, San Diego, CA, Feb. 2011.
[3] - "Modeling and analysis of K-tier downlink heterogeneous cellular networks," IEEE J. Sel. Areas Commun., vol. 30, no. 3, pp. 550-560, Apr. 2012.

[4] H. Keeler, B. Blaszczyszyn, and M. Karray, "SINR-based k-coverage probability in cellular networks with arbitrary shadowing," in Proc. of IEEE ISIT, Istanbul, Turkey, Jul. 2013, pp. 1167-1171.

[5] Y. Kim, S. Lee, and D. Hong, "Performance analysis of two-tier femtocell networks with outage constraints," IEEE Trans. Wireless Commun., vol. 9, no. 9, pp. 2695-2700, Sep. 2010.

[6] H. S. Dhillon, R. K. Ganti, and J. G. Andrews, "Load-aware heterogeneous cellular networks: Modeling and SIR distribution," in Proc. of IEEE GLOBECOM, Anaheim, CA, Dec. 2012, pp. 4314-4319.

[7] S. Singh, H. S. Dhillon, and J. G. Andrews, "Offloading in heterogeneous networks: Modeling, analysis and design insights," IEEE Trans. Wireless Commun., vol. 12, no. 5, pp. 2484-2497, May 2013.

[8] H.-S. Jo, P. Xia, and J. G. Andrews, "Open, closed, and shared access femtocells in the downlink," EURASIP J. on Wireless Commun. and Netw., vol. 2012, no. 363, Dec. 2012.

[9] F. Baccelli and A. Giovanidis, "A stochastic geometry framework for analyzing pairwise-cooperative cellular networks," IEEE Trans. Wireless Commun., vol. 14, no. 2, pp. 794-808, Feb. 2015.

[10] G. Nigam, P. Minero, and M. Haenggi, "Coordinated multipoint join$\mathrm{t}$ transmission in heterogeneous networks," IEEE Trans. Commun., vol. 62, no. 11, pp. 4134-4146, Nov. 2014.

[11] S. Kishore, L. Greenstein, H. Poor, and S. Schwartz, "Uplink user capacity in a CDMA macrocell with a hotspot microcell: exact and approximate analyses," IEEE Trans. Wireless Commun., vol. 2, no. 2, pp. 364-374, Mar. 2003.

[12] — "Uplink user capacity in a multicell CDMA system with hotspot microcells," IEEE Trans. Wireless Commun., vol. 5, no. 6, pp. 13331342, June 2006.

[13] X. An and F. Pianese, "Understanding co-channel interference in LTEbased multi-tier cellular networks," in Proc. of ACM PE-WASUN, Paphos, Cyprus, Oct. 2012, pp. 107-112.

[14] T. D. Novlan, H. S. Dhillon, and J. G. Andrews, "Analytical modeling of uplink cellular networks," IEEE Trans. Wireless Commun., vol. 12, no. 6, pp. 1536-1276, Jun. 2013.

[15] T. Novlan and J. Andrews, "Analytical evaluation of uplink fractional frequency reuse," IEEE Trans. Wireless Commun., vol. 61, no. 5, pp. 2098-2108, May 2013.

[16] N. Chakchouk and B. Hamdaoui, "Uplink performance characterization and analysis of two-tier femtocell networks," IEEE Trans. Veh. Technol., vol. 61, no. 9, pp. 4057-4068, Nov. 2012.

[17] V. Chandrasekhar and J. Andrews, "Uplink capacity and interference avoidance for two-tier femtocell networks," IEEE Trans. Wireless Commun., vol. 8, no. 7, pp. 3498-3509, Jul. 2009.

[18] W. C. Cheung, T. Q. S. Quek, and M. Kountouris, "Stochastic analysis of two-tier networks: Effect of spectrum allocation," in Proc. of IEEE ICASSP, Prague, Czech Republic, May 2011, pp. 2964-2967.

[19] W. Bao and B. Liang, "Uplink interference analysis for two-tier cellular networks with diverse users under random spatial patterns," IEEE Trans. Wireless Commun., vol. 14, no. 3, pp. 1252-1265, Mar. 2015.

[20] P. Xia, V. Chandrasekhar, and J. Andrews, "Open vs. closed access femtocells in the uplink," IEEE Trans. Wireless Commun., vol. 9, no. 12, pp. 3798-3809, Dec. 2010.

[21] P. Tarasak, T. Q. S. Quek, and F. P. S. Chin, "Uplink timing misalignment in open and closed access OFDMA femtocell networks," IEEE Commun. Lett., vol. 15, no. 9, pp. 926-928, Sep. 2011.

[22] Z. Zeinalpour-Yazdi and S. Jalali, "Outage analysis of uplink two-tier networks," IEEE Trans. on Communications, vol. 62, no. 9, pp. 33513362, Sep. 2014.

[23] H. ElSawy and E. Hossain, "On stochastic geometry modeling of cellular uplink transmission with truncated channel inversion power control," IEEE Trans. Wireless Commun., vol. 13, no. 8, pp. 4454-4469, Aug. 2014.

[24] S. Singh, X. Zhang, and J. G. Andrews, "Joint rate and SINR coverage analysis for decoupled uplink-downlink biased cell associations in HetNets," arXiv:1412.1898 [cs.IT].

[25] M. Y. Arslan, J. Yoon, K. Sundaresan, S. V. Krishnamurthy, and S. Banerjee, "FERMI: a femtocell resource management system forinterference mitigation in OFDMA networks," in Proc. of ACM MobiCom, Las Vegas, NV, Sep. 2011, pp. 25-36.

[26] J. Yoon, M. Y. Arslan, K. Sundaresan, S. V. Krishnamurthy, and S. Banerjee, "A distributed resource management framework for interference mitigation in OFDMA femtocell networks," in Proc. of ACM MobiHoc, Hilton Head Island, SC, Jun. 2012, pp. 233-242. 
[27] W. Bao and B. Liang, "Understanding the benefits of open access in femtocell networks: Stochastic geometric analysis in the uplink," in Proc. of ACM/IEEE MSWiM, Barcelona, Spain, Nov. 2013, pp. 237246.

[28] C. C. Chan and S. Hanly, "Calculating the outage probability in a CDMA network with spatial Poisson traffic," Trans. Veh. Technol., vol. 50, no. 1, pp. 183-204, Jan. 2001

[29] Q. Ye, M. Al-Shalash, C. Caramanis, and J. Andrews, "Resource optimization in device-to-device cellular systems using time-frequency hopping," IEEE Trans. Wireless Commun., vol. 13, no. 10, pp. 54675480, Oct. 2014.

[30] D. Stoyan, W. Kendall, and J. Mecke, Stochastic Geometry and Its Applications, 2nd ed. Wiley, 1995.

[31] H.-S. Jo, Y. J. Sang, P. Xia, and J. G. Andrews, "Heterogeneous cellular networks with flexible cell association: A comprehensive downlink SINR analysis," IEEE Trans. Wireless Commun., vol. 11, no. 10, pp. 3484 3495, Oct. 2012.

[32] K. Gilhousen, I. Jacobs, R. Padovani, A. Viterbi, L. Weaver, and C. Wheatley, "On the capacity of a cellular CDMA system," IEEE Trans. Veh. Technol., vol. 40, no. 2, pp. 303-312, May 1991.

[33] A. J. Viterbi, A. M. Viterbi, K. Gilhousen, and E. Zehavi, "Soft handoff extends CDMA cell coverage and increases reverse link capacity," IEEE J. Sel. Areas Commun., vol. 12, no. 8, pp. 1281-1288, Oct. 1994

[34] F. Baccelli and B. Blaszczyszyn, "Stochastic geometry and wireless networks, volume 1: Theory," Foundations and Trends in Networking, vol. 3, no. 3-4, pp. 249-449, 2009.

[35] 3GPP-TR-36.814, "Evolved universal terrestrial radio access (E-UTRA); further advancements for E-UTRA physical layer aspects (release 9)," Mar. 2010.

[36] W. Bao and B. Liang, "Handoff rate analysis in heterogeneous cellular networks: a stochastic geometric approach," in Proc. of ACM MSWiM, Montreal, Canada, Sep. 2014, pp. 95-102.

[37] — "Stochastic geometric analysis of user mobility in heterogeneous wireless networks," to appear in IEEE J. Sel. Areas Commun., Oct. 2015.

[38] — , "Handoff rate analysis in heterogeneous wireless networks with Poisson and Poisson cluster patterns," in Proc. of ACM MobiHoc, Hangzhou, China, Jun. 2015, pp. 77-86.

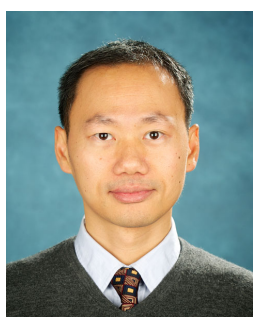

Ben Liang (S'94-M'01-SM'06) received honorssimultaneous B.Sc. (valedictorian) and M.Sc. degrees in Electrical Engineering from Polytechnic University in Brooklyn, New York, in 1997 and the Ph.D. degree in Electrical Engineering with Computer Science minor from Cornell University in Ithaca, New York, in 2001. In the 2001 - 2002 academic year, he was a visiting lecturer and postdoctoral research associate at Cornell University. $\mathrm{He}$ joined the Department of Electrical and Computer Engineering at the University of Toronto in 2002 , where he is now a Professor. His current research interests are in mobile communications and networked systems. He has served as an Editor for IEEE TRANSACTIONS ON COMMUNICATIONS, as an Editor for IEEE TRANSACTIONS ON WIRELESS COMMUNICATIONS, and as an Associate Editor for the Wiley SECURITY AND COMMUNICATION NETWORKS journal, in addition to regularly serving on the organizational and technical committees of a number of conferences. He is a senior member of IEEE and a member of ACM and Tau Beta Pi.

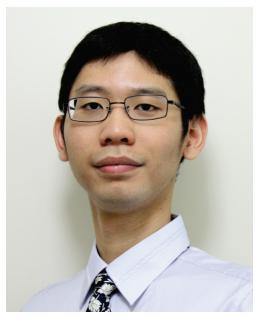

Wei Bao (S'10) received the B.E. degree in Communications Engineering from the Beijing University of Posts and Telecommunications, Beijing, China, in 2009, and the M.A.Sc. degree in Electrical and Computer Engineering from the University of British Columbia, Vancouver, BC, Canada, in 2011. He is currently a Ph.D. student at the Department of Electrical and Computer Engineering, University of Toronto, Toronto, ON, Canada. His research interests are stochastic and queueing networks, stochastic geometry, and performance analysis of heterogeneous wireless networks. He received the Ontario Trillium Scholarship in 2011, and the Best Paper Award in ACM International Conference on Modeling, Analysis and Simulation of Wireless and Mobile Systems (MSWiM) in 2013. 